\title{
MULTILINEAR COMMUTATORS FOR FRACTIONAL INTEGRALS IN NON-HOMOGENEOUS SPACES
}

\author{
Guoen Hu, Yan Meng and Dachung Yang*
}

Abstract

Under the assumption that $\mu$ is a non-doubling measure on $\mathbb{R}^{d}$, the authors obtain the $\left(L^{p}, L^{q}\right)$-boundedness and the weak type endpoint estimate for the multilinear commutators generated by fractional integrals with $\operatorname{RBMO}(\mu)$ functions of Tolsa or with

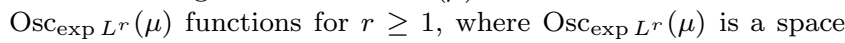
of Orlicz type satisfying that $\operatorname{Osc}_{\exp } L^{r}(\mu)=\operatorname{RBMO}(\mu)$ if $r=1$ and $\mathrm{Osc}_{\exp L^{r}}(\mu) \subset \operatorname{RBMO}(\mu)$ if $r>1$.

\section{Introduction}

Let $\mu$ be a positive Radon measure on $\mathbb{R}^{d}$ which only satisfies the following growth condition

$$
\mu(B(x, r)) \leq C r^{n}
$$

for all $x \in \mathbb{R}^{d}$ and $r>0$, where $n$ is a fixed number and $0<n \leq d$. The doubling condition on $\mu$, namely, there exists some positive constant $C$ such that

$$
\mu(B(x, 2 r)) \leq C \mu(B(x, r))
$$

for all $x \in \operatorname{supp} \mu$ and $r>0$, is an essential assumption in the classical theory of harmonic analysis. But recently, many classical results have been proved still valid if the underlying measure $\mu$ is substituted by a non-doubling Radon measure as in (1.1); see $[\mathbf{1 0}],[\mathbf{1 7}],[\mathbf{1 8}],[\mathbf{1 1}],[\mathbf{1 9}]$, $[\mathbf{2 0}],[\mathbf{2 1}],[\mathbf{2 2}],[\mathbf{2 3}],[\mathbf{1 2}],[\mathbf{1 3}],[\mathbf{8}],[\mathbf{6}],[\mathbf{1 4}],[\mathbf{2}],[\mathbf{4}],[\mathbf{5}],[\mathbf{7}]$ and their references.

The purpose of this paper is to prove the $\left(L^{p}, L^{q}\right)$-boundedness and the weak type estimate for any multilinear commutator generated by the fractional integral $I_{\alpha}$ related to a measure $\mu$ as in (1.1) for $0<\alpha<n$

2000 Mathematics Subject Classification. Primary: 47B47; Secondary: 42B20, 42B25, 47A30.

Key words. Non-doubling measure, fractional integral, commutator, Lebesgue space, endpoint estimate, $\mathrm{RBMO}(\mu)$, Osc $\exp ^{r}(\mu)$.

${ }^{*}$ Corresponding author. 
with any $\operatorname{RBMO}(\mu)$ function of Tolsa in $[\mathbf{2 0}]$ or with any $\operatorname{Osc}_{\exp L^{r}}(\mu)$ function for $r \geq 1$, motivated by [16], [3] for commutators on $\mathbb{R}^{d}$ in the case that $\mu$ is the $d$-dimensional Lebesgue measure there. Here, for $0<\alpha<n$ and all $x \in \operatorname{supp}(\mu)$,

$$
I_{\alpha} f(x)=\int_{\mathbb{R}^{d}} \frac{1}{|x-y|^{n-\alpha}} f(y) d \mu(y) .
$$

The behavior of such fractional integrals on a metric space was recently studied by García-Cuerva and Gatto in [4]. Chen and Sawyer [2] showed that the first order commutator generated by $I_{\alpha}$ and $\operatorname{RBMO}(\mu)$ function enjoys the same $\left(L^{p}, L^{q}\right)$ mapping properties as in the case that $\mu$ is the Lebesgue measure. However, it seems that the argument used in [2] does not apply to the multilinear commutator here, we will employ some ideas used in [2] and some new ideas different from ones used in [2].

Before stating our results, let us introduce some notation and recall some definitions. Throughout this paper, we only consider closed cubes with sides parallel to coordinate axes. Let $\alpha>1$ and $\beta>\alpha^{n}$. We say that a cube $Q$ is a $(\alpha, \beta)$-doubling cube if $\mu(\alpha Q) \leq \beta \mu(Q)$, where $\alpha Q$ denotes the cube with the same center as $Q$ and $l(\alpha Q)=\alpha l(Q)$. In what follows, for definiteness, if $\alpha$ and $\beta$ are not specified, by a doubling cube we mean a $\left(2,2^{d+1}\right)$-doubling cube. Especially, for any given cube $Q$, we denote by $\widetilde{Q}$ the smallest doubling cube which contains $Q$ and has the same center as $Q$.

Let $0 \leq \gamma<n$. Given two cubes $Q \subset R$ in $\mathbb{R}^{d}$, set

$$
K_{Q, R}^{(\gamma)}=1+\sum_{k=1}^{N_{Q, R}}\left[\frac{\mu\left(2^{k} Q\right)}{l\left(2^{k} Q\right)^{n}}\right]^{1-\gamma / n},
$$

where $N_{Q, R}$ is the smallest positive integer $k$ such that $l\left(2^{k} Q\right) \geq l(R)$. If $\gamma=0$, then we denote $K_{Q, R}^{(0)}$ by $K_{Q, R}$. Tolsa in [20] first introduced the concept of $K_{Q, R}$ and gave its several useful properties. Chen and Sawyer in [2] introduced $K_{Q, R}^{(\gamma)}$ and established some properties on $K_{Q, R}^{(\gamma)}$ similar to those on $K_{Q, R}$.

Using $K_{Q, R}$, Tolsa in [20] introduced the space $\operatorname{RBMO}(\mu)$ with the non-doubling measure $\mu$, which is proved to be a good substitute of the classical space BMO in this case.

Definition 1.1. Let $\rho>1$ be some fixed constant. We say that a function $f \in L_{\mathrm{loc}}^{1}(\mu)$ is in $\operatorname{RBMO}(\mu)$ if there exists some constant $C>0$ such that for any cube $Q$ centered at some point of $\operatorname{supp}(\mu)$,

$$
\frac{1}{\mu(\rho Q)} \int_{Q}\left|f(y)-m_{\widetilde{Q}}(f)\right| d \mu(y) \leq C
$$


and

$$
\left|m_{Q}(f)-m_{R}(f)\right| \leq C K_{Q, R}
$$

for any two doubling cube $Q \subset R$, where $m_{Q}(f)$ denotes the mean of $f$ over cube $Q$. The minimal constant $C$ as above is the $\operatorname{RBMO}(\mu)$ norm of $f$ and is denoted by $\|f\|_{*}$.

It has been shown in $[\mathbf{2 0}]$ that the definition of $\operatorname{RBMO}(\mu)$ is independent of chosen constant $\rho$. In this paper, we need to choose different $\rho$ in the proof of Theorem 1.1 and Theorem 1.2 below, respectively.

For any $m \in \mathbb{N}, 0<\alpha<n$ and $b_{i} \in \operatorname{RBMO}(\mu), i=1,2, \ldots, m$, the multilinear commutator, $I_{\alpha ; b_{1}, \ldots, b_{m}}$, is defined by $\left[b_{m}, \ldots,\left[b_{2},\left[b_{1}, I_{\alpha}\right]\right] \ldots\right]$, that is,

$$
I_{\alpha ; b_{1}, b_{2}, \ldots, b_{m}} f(x)=\int_{\mathbb{R}^{d}} \prod_{j=1}^{m}\left[b_{j}(x)-b_{j}(y)\right] \frac{f(y)}{|x-y|^{n-\alpha}} d \mu(y) .
$$

When $m=1$, the operator $I_{\alpha ; b_{1}, \ldots, b_{m}}$ is just the commutator $\left[b_{1}, I_{\alpha}\right]$, which is a variant on the non-doubling measure of the classical commutator studied by Chanillo in [1]. In [16], Pérez and Trujillo-González studied the boundedness of multilinear operators of this type generated by Calderón-Zygmund operators with $\mathrm{BMO}\left(\mathbb{R}^{d}\right)$ functions or with $\mathrm{Osc}_{\exp } L^{r}$ functions for $r \geq 1$ in the case that $\mu$ is the $d$-dimensional Lebesgue measure. An extensive study of multilinear operators of this type can also be founded in $[\mathbf{9}]$. In $[\mathbf{7}]$, the authors obtained the corresponding results of multilinear operators generated by Calderón-Zygmund operators with $\operatorname{RBMO}(\mu)$ functions or with $\operatorname{Osc}_{\exp L^{r}}(\mu)$ functions for $r \geq 1$ in the case that $\mu$ is a non doubling measure. The multilinear commutator $I_{\alpha ; b_{1}, \ldots, b_{m}}$ can be regarded as a natural variant of these multilinear operators in $[\mathbf{9}],[\mathbf{1 6}],[\mathbf{7}]$.

Chen and Sawyer in [2] proved that $I_{\alpha ; b_{1}}$ as in (1.2) is bounded from $L^{p}(\mu)$ to $L^{q}(\mu)$ provided that $1<p<n / \alpha$ and $1 / q=1 / p-\alpha / n$. For $m \geq 2$, a conclusion similar to that for the case $m=1$ in $[2]$ can be obtained as follows.

Theorem 1.1. Let $m \in \mathbb{N}$ and $b_{j} \in \operatorname{RBMO}(\mu), j=1,2, \ldots, m$. For $\alpha \in$ $(0, n)$, let $I_{\alpha ; b_{1}, b_{2}, \ldots, b_{m}}$ be as in (1.2). Then there exists a constant $C>0$ such that for all $f \in L^{p}(\mu)$,

$$
\left\|I_{\alpha ; b_{1}, b_{2}, \ldots, b_{m}} f\right\|_{L^{q}(\mu)} \leq C \prod_{j=1}^{m}\left\|b_{j}\right\|_{*}\|f\|_{L^{p}(\mu)},
$$

where $1<p<n / \alpha$ and $1 / q=1 / p-\alpha / n$. 
Remark 1.1. It is well-known that the commutator $\left[b, I_{\alpha}\right]$ is a bounded operator from $L^{p}\left(\mathbb{R}^{d}\right)$ to $L^{q}\left(\mathbb{R}^{d}\right)$ provided $1<p<d / \alpha$ and $1 / q=1 / p-$ $\alpha / d$, if and only if $b \in \mathrm{BMO}\left(\mathbb{R}^{d}\right)$ if the measure $\mu$ is the $d$-dimensional Lebesgue measure; see [1]. However, it is still open if $b \in \operatorname{RBMO}(\mu)$ is a necessary condition for the $\left(L^{p}(\mu), L^{q}(\mu)\right)$-boundedness of the multilinear commutators $I_{\alpha ; b_{1}, \ldots, b_{m}}$ on non doubling measures.

To consider the endpoint case of Theorem 1.1, we introduce the following function space, which is a variant with a non-doubling measure of the space $\operatorname{Osc}_{\exp } L^{r}$ in $[\mathbf{1 6}]$.

Definition 1.2. For $r \geq 1$, a locally integrable function $f$ is said to belong to the space $\operatorname{Osc}_{\exp L^{r}}(\mu)$ if there is a constant $C_{1}>0$ such that

(i) for any $Q$,

$$
\begin{aligned}
& \left\|f-m_{\widetilde{Q}}(f)\right\|_{\exp L^{r}, Q, \mu / \mu(2 Q)} \\
= & \inf \left\{\lambda>0: \frac{1}{\mu(2 Q)} \int_{Q} \exp \left(\frac{\left|f(x)-m_{\widetilde{Q}}(f)\right|}{\lambda}\right)^{r} d \mu(x) \leq 2\right\} \leq C_{1},
\end{aligned}
$$

(ii) for any doubling cubes $Q_{1} \subset Q_{2}$,

$$
\left|m_{Q_{1}}(f)-m_{Q_{2}}(f)\right| \leq C_{1} K_{Q_{1}, Q_{2}} .
$$

The minimal constant $C_{1}$ satisfying (i) and (ii) is the $\operatorname{Osc}_{\exp L^{r}}(\mu)$ norm

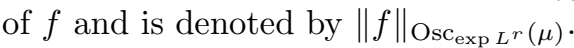

Obviously, for any $r \geq 1, \operatorname{Osc}_{\exp L^{r}(\mu)} \subset \operatorname{RBMO}(\mu)$. Moreover, from John-Nirenberg's inequality in [20] (see also Lemma 3.1 below), it follows that $\mathrm{Osc}_{\exp L(\mu)}=\operatorname{RBMO}(\mu)$. We remark that it was pointed by Pérez and Trujillo-González in $[\mathbf{1 6}]$ that if $\mu$ is the $d$-dimensional Lebesgue measure in $\mathbb{R}^{d}$, the counterpart in $[\mathbf{1 6}]$ of the space $\operatorname{Osc}_{\exp L^{r}}(\mu)$ when $r>1$ is a proper subspace of the classical space $\operatorname{BMO}\left(\mathbb{R}^{d}\right)$. However, it is still unknown if the space $\operatorname{Osc}_{\exp L^{r}}(\mu)$ is a proper subspace of the space $\operatorname{RBMO}(\mu)$ when $\mu$ is a non-doubling measure?

To state the weak type estimate for the multilinear commutator $I_{\alpha ; b_{1}, \ldots, b_{m}}$, we still need to introduce the following notation. For $1 \leq j \leq m$, we denote by $C_{j}^{m}$ the family of all finite subset $\sigma=\{\sigma(1), \ldots, \sigma(j)\}$ of $\{1,2, \ldots, m\}$ with $j$ different elements. For any $\sigma \in C_{j}^{m}$, the complementary sequence $\sigma^{\prime}$ is given by $\sigma^{\prime}=\{1,2, \ldots, m\} \backslash \sigma$. For any $\sigma=$ $\{\sigma(1), \sigma(2), \ldots, \sigma(j)\} \in C_{j}^{m}$, we write for any $m$-tuple $r=\left(r_{1}, r_{2}, \ldots, r_{m}\right)$, $1 / r_{\sigma}=1 / r_{\sigma(1)}+\cdots+1 / r_{\sigma(j)}$ and $1 / r_{\sigma^{\prime}}=1 / r-1 / r_{\sigma}$, where $1 / r=$ $1 / r_{1}+\cdots+1 / r_{m}$ 
The following weak type estimate is the main result of this paper, which is new even when $m=1$, namely, Theorem 1.2 is also new even for the commutator of the first order, $\left[b_{1}, I_{\alpha}\right]$.

Theorem 1.2. Let $0<\alpha<n, q_{0}=n /(n-\alpha), m \in \mathbb{N}, r_{i} \geq 1$ and $b_{i} \in \mathrm{Osc}_{\exp L^{r_{i}}(\mu)}$ for $i=1,2, \ldots, m$. Let $I_{\alpha ; b_{1}, \ldots, b_{m}}$ be as in (1.2). Then there exists a constant $C>0$ such that for all bounded functions $f$ with compact support and all $\lambda>0$,

$$
\begin{aligned}
& \mu\left(\left\{x \in \mathbb{R}^{d}:\left|I_{\alpha ; b_{1}, \ldots, b_{m}} f(x)\right|>\lambda\right\}\right) \\
& \leq C {\left[\Phi_{1 / r}\left(\prod_{j=1}^{m}\left\|b_{j}\right\|_{\mathrm{Osc}_{\exp L^{r_{j}}}(\mu)}\right)\right]^{q_{0}} } \\
& \times\left\{\sum_{j=0}^{m} \sum_{\sigma \in C_{j}^{m}} \Phi_{1 / r_{\sigma}}\left[\left\|\Phi_{1 / r_{\sigma^{\prime}}}\left(\lambda^{-1}|f|\right)\right\|_{L^{1}(\mu)}\right]\right\}^{q_{0}},
\end{aligned}
$$

where $\Phi_{s}(t)=t \log ^{s}(2+t)$ for all $t>0$ and $s>0$.

In what follows, $C$ denotes a constant that is independent of main parameters involved but whose value may differ from line to line. For any index $p \in[1, \infty]$, we denote by $p^{\prime}$ its conjugate index, namely, $1 / p+$ $1 / p^{\prime}=1$. For $A \sim B$, we mean that there is a constant $C>0$ such that $C^{-1} B \leq A \leq C B$.

Let $1 \leq s<\infty$. For a $\mu$-locally integrable function $f$ and a cube $Q$, we define

$$
\begin{aligned}
& \|f\|_{L \log ^{s} L, Q, \mu / \mu(2 Q)} \\
& \quad=\inf \left\{\lambda>0: \frac{1}{\mu(2 Q)} \int_{Q} \frac{|f(x)|}{\lambda} \log ^{s}\left(2+\frac{|f(x)|}{\lambda}\right) d \mu(x) \leq 1\right\}
\end{aligned}
$$

and

$\|f\|_{\exp L^{s}, Q, \mu / \mu(2 Q)}=\inf \left\{\lambda>0: \frac{1}{\mu(2 Q)} \int_{Q} \exp \left(\frac{|f(x)|}{\lambda}\right)^{s} d \mu(x) \leq 2\right\}$.

Then the generalized Hölder inequality

$$
\frac{1}{\mu(2 Q)} \int_{Q}\left|f(x) b_{1}(x) \cdots b_{m}(x)\right| d \mu(x)
$$

$$
\leq C\|f\|_{L \log ^{1 / r} L, Q, \mu / \mu(2 Q)}\left\|b_{1}\right\|_{\exp L^{r_{1}, Q, \mu / \mu(2 Q)}} \cdots\left\|b_{m}\right\|_{\exp L^{r_{m}}, Q, \mu / \mu(2 Q)}
$$


holds for $\mu$-locally integrable functions $f$ and $b_{i}, i=1,2, \ldots, m$, and any cube $Q$, provided that $r_{1}, r_{2}, \ldots, r_{m} \geq 1,1 / r=1 / r_{1}+\cdots+1 / r_{m}$. If $\mu$ is the $d$-dimensional Lebesgue measure, (1.3) was proved in [16]. It is easy to see that the proof in $[\mathbf{1 6}]$ still works in non-homogeneous spaces.

\section{Proof of Theorem 1.1}

Before we begin to prove Theorem 1.1, we state one equivalent norm for the space $\operatorname{RBMO}(\mu)$ and some lemmas which play important roles in the proof.

Let $\rho>1$. For any given function $b \in \operatorname{RBMO}(\mu)$, there exists some constant $C_{2}>0$ and a collection of numbers $\left\{b_{Q}\right\}_{Q}$, namely, for each cube $Q$, there exists a number $b_{Q} \in \mathbb{R}$, such that

$$
\sup _{Q} \frac{1}{\mu(\rho Q)} \int_{Q}\left|b(y)-b_{Q}\right| d \mu(y) \leq C_{2}
$$

and

$$
\left|b_{Q}-b_{R}\right| \leq C_{2} K_{Q, R}
$$

for any two cubes $Q \subset R$. Let $\|b\|_{* *}=\inf \left\{C_{2}\right\}$, where the infimum is taken over all $C_{2}>0$ as above. Then there is a constant $C>0$ such that for all $b \in \operatorname{RBMO}(\mu)$,

$$
C^{-1}\|b\|_{*} \leq\|b\|_{* *} \leq C\|b\|_{*} ;
$$

see $[\mathbf{2 0}]$.

Lemma $2.1([6],[4])$. Let $1<p<\infty, 1 / q=1 / p-\alpha / n$ and $0<\alpha<n$. Then

$$
\left\|I_{\alpha} f\right\|_{L^{q}(\mu)} \leq C\|f\|_{L^{p}(\mu)} .
$$

Lemma $2.2([\mathbf{2}])$. For $\eta>1$ and $0 \leq \beta<n / p$, we define the non centered maximal operator

$$
M_{p,(\eta)}^{(\beta)} f(x)=\sup _{x \in Q}\left[\frac{1}{\mu(\eta Q)^{1-\beta p / n}} \int_{Q}|f(y)|^{p} d \mu(y)\right]^{1 / p} .
$$

When $\beta=0$, we denote $M_{p,(\eta)}^{(\beta)}$ simply by $M_{p,(\eta)}$. If $p<r<n / \beta$ and $1 / q=1 / r-\beta / n$, then

$$
\left\|M_{p,(\eta)}^{(\beta)} f\right\|_{L^{q}(\mu)} \leq C\|f\|_{L^{r}(\mu)},
$$

and if $q>p$, then

$$
\left\|M_{p,(\eta)} f\right\|_{L^{q}(\mu)} \leq C\|f\|_{L^{q}(\mu)} .
$$


Moreover, we also need to introduce another variant sharp maximal operator $M^{\sharp,(\beta)} f$ defined by

$$
\begin{aligned}
M^{\sharp,(\beta)} f(x)=\sup _{x \in Q} \frac{1}{\mu\left(\frac{3}{2} Q\right)} \int_{Q} \mid f(y)- & m_{\widetilde{Q}}(f) \mid d \mu(y) \\
& +\sup _{\substack{x \in Q \subset R \\
Q, R \text { doubling }}} \frac{\left|m_{Q}(f)-m_{R}(f)\right|}{K_{Q, R}^{(\beta)}},
\end{aligned}
$$

and non centered doubling maximal operator $N$ :

$$
N f(x)=\sup _{\substack{x \in Q \\ Q \text { doubling }}} \frac{1}{\mu(Q)} \int_{Q}|f(y)| d \mu(y) .
$$

By the Lebesgue differentiation theorem, it is easy to see that for any $f \in L_{\mathrm{loc}}^{1}(\mu)$,

$$
|f(x)| \leq N f(x)
$$

for $\mu$-a.e. $x \in \mathbb{R}^{d}$; see $[\mathbf{2 0}]$ and $[\mathbf{2}]$.

Lemma $2.3([\mathbf{2}])$. Let $f \in L_{l o c}^{1}(\mu)$ with

$$
\int_{\mathbb{R}^{d}} f(y) d \mu(y)=0
$$

if $\|\mu\|<\infty$. For $1<p<\infty$, if $\inf (1, N f) \in L^{p}(\mu)$, then for $0 \leq \beta<n$, we have

$$
\|N f\|_{L^{p}(\mu)} \leq C\left\|M^{\sharp,(\beta)} f\right\|_{L^{p}(\mu)} .
$$

Proof of Theorem 1.1: For simplicity, we only consider the case of $m=2$. If $m \geq 2$, we can deduce the conclusion of the theorem by induction on $m$. We leave the details to the reader; see also the proof of Theorem 1.2 below.

For all $r \in(1, n / \alpha)$, we will prove the following sharp maximal function estimate

$$
\begin{aligned}
& M^{\sharp,(\alpha)}\left(I_{\alpha ; b_{1}, b_{2}} f\right)(x) \leq C\left\|b_{1}\right\|_{*}\left\|b_{2}\right\|_{*}\left\{M_{r,(9 / 8)}^{(\alpha)} f(x)\right. \\
&+\left.M_{r,(3 / 2)}\left[I_{\alpha}(|f|)\right](x)\right\} \\
&+C\left\|b_{1}\right\|_{*} M_{r,(3 / 2)}\left(I_{\alpha ; b_{2}} f\right)(x) \\
&+C\left\|b_{2}\right\|_{*} M_{r,(3 / 2)}\left(I_{\alpha ; b_{1}} f\right)(x) .
\end{aligned}
$$


Then choose $r$ such that $1<r<p<n / \alpha$ and $1 / q=1 / p-\alpha / n$. By (2.3), (2.2), Lemma 2.1, Lemma 2.2 and Lemma 2.3, we obtain

$$
\begin{aligned}
\left\|I_{\alpha ; b_{1}, b_{2}} f\right\|_{L^{q}(\mu)} \leq & C\left\|N\left(I_{\alpha ; b_{1}, b_{2}} f\right)\right\|_{L^{q}(\mu)} \\
\leq & C\left\|M^{\sharp,(\alpha)}\left(I_{\alpha ; b_{1}, b_{2}} f\right)\right\|_{L^{q}(\mu)} \\
\leq & C\left\|b_{1}\right\|_{*}\left\|b_{2}\right\|_{*}\left\{\left\|M_{r,(9 / 8)}^{(\alpha)} f\right\|_{L^{q}(\mu)}\right. \\
& \left.+\left\|M_{r,(3 / 2)}\left[I_{\alpha}(|f|)\right]\right\|_{L^{q}(\mu)}\right\} \\
& +C\left\|b_{1}\right\|_{*}\left\|M_{r,(3 / 2)}\left(I_{\alpha ; b_{2}} f\right)\right\|_{L^{q}(\mu)} \\
& +C\left\|b_{2}\right\|_{*}\left\|M_{r,(3 / 2)}\left(I_{\alpha ; b_{1}} f\right)\right\|_{L^{q}(\mu)} \\
\leq & C\left\|b_{1}\right\|_{*}\left\|b_{2}\right\|_{*}\|f\|_{L^{p}(\mu)},
\end{aligned}
$$

which is the desired conclusion.

For $j=1,2$, let $\left\{b_{Q}^{j}\right\}_{Q}$ be a family of numbers satisfying

$$
\int_{Q}\left|b_{j}(y)-b_{Q}^{j}\right| d \mu(y) \leq 2 \mu(2 Q)\left\|b_{j}\right\|_{* *}
$$

for any cube $Q$, and

$$
\left|b_{Q}^{j}-b_{R}^{j}\right| \leq 2 K_{Q, R}\|b\|_{* *}
$$

for all cubes $Q \subset R$. For any cube $Q$, we let

$$
h_{Q}=m_{Q}\left(I_{\alpha}\left[\left(b_{1}-b_{Q}^{1}\right)\left(b_{2}-b_{Q}^{2}\right) f \chi_{\mathbb{R}^{d} \backslash \frac{4}{3} Q}\right]\right) .
$$

To establish (2.3), it suffices to verify that

$$
\begin{aligned}
& \frac{1}{\mu\left(\frac{3}{2} Q\right)} \int_{Q}\left|I_{\alpha ; b_{1}, b_{2}} f(y)-h_{Q}\right| d \mu(y) \\
& \leq C\left\|b_{1}\right\|_{*}\left\|b_{2}\right\|_{*}\left[M_{r,(9 / 8)}^{(\alpha)} f(x)+M_{r,(3 /)}\left(I_{\alpha} f\right)(x)\right] \\
& \quad+C\left\|b_{1}\right\|_{*} M_{r,(3 / 2)}\left(I_{\alpha ; b_{2}} f\right)(x)+C\left\|b_{2}\right\|_{*} M_{r,(3 / 2)}\left(I_{\alpha ; b_{1}} f\right)(x)
\end{aligned}
$$


holds for any cube $Q$ and any $x \in Q$, and

$$
\begin{aligned}
\left|h_{Q}-h_{R}\right| \leq & C\left\|b_{1}\right\|_{*}\left\|b_{2}\right\|_{*} K_{Q, R}^{2}\left\{K_{Q, R}^{(\alpha)} M_{r,(9 / 8)}^{(\alpha)} f(x)\right. \\
& \left.+M_{r,(3 / 2)}\left[I_{\alpha}(|f|)\right](x)\right\} \\
& +C\left\|b_{1}\right\|_{*} K_{Q, R} M_{r,(3 / 2)}\left(I_{\alpha ; b_{2}} f\right)(x) \\
& +C\left\|b_{2}\right\|_{*} K_{Q, R} M_{r,(3 / 2)}\left(I_{\alpha ; b_{1}} f\right)(x)
\end{aligned}
$$

for any cubes $Q \subset R$ with $x \in Q$, where $Q$ is an arbitrary cube and $R$ is a doubling cube.

By a method similar to that in [2], from (2.4) and (2.5), it is then easy to deduce the sharp maximal function estimate (2.3).

To obtain the estimate (2.4) for any fixed $Q$ and $x \in Q$, write

$$
\begin{aligned}
& \frac{1}{\mu\left(\frac{3}{2} Q\right)} \int_{Q}\left|I_{\alpha ; b_{1}, b_{2}} f(y)-h_{Q}\right| d \mu(y) \\
& \leq \frac{1}{\mu\left(\frac{3}{2} Q\right)} \int_{Q} \prod_{j=1,2}\left|b_{j}(y)-b_{Q}^{j}\right|\left|I_{\alpha} f(y)\right| d \mu(y) \\
&+\frac{1}{\mu\left(\frac{3}{2} Q\right)} \int_{Q}\left|b_{1}(y)-b_{Q}^{1}\right|\left|I_{\alpha ; b_{2}} f(y)\right| d \mu(y) \\
&+\frac{1}{\mu\left(\frac{3}{2} Q\right)} \int_{Q}\left|b_{2}(y)-b_{Q}^{2}\right|\left|I_{\alpha ; b_{1}} f(y)\right| d \mu(y) \\
&+\frac{1}{\mu\left(\frac{3}{2} Q\right)} \int_{Q}\left|I_{\alpha}\left[\left(b_{1}-b_{Q}^{1}\right)\left(b_{1}-b_{Q}^{1}\right) f\right](y)-h_{Q}\right| d \mu(y) \\
&= \mathrm{I}_{1}+\mathrm{I}_{2}+\mathrm{I}_{3}+\mathrm{I}_{4} .
\end{aligned}
$$

The Hölder inequality and Corollary 3.5 in $[\mathbf{2 0}]$ yield that for $1<r<$ $n / \alpha$,

$$
\begin{aligned}
\mathrm{I}_{1} \leq\left\{\frac{1}{\mu\left(\frac{3}{2} Q\right)} \int_{Q}\left|\left(b_{1}(y)-b_{Q}^{1}\right)\left(b_{2}(y)-b_{Q}^{2}\right)\right|^{r^{\prime}} d \mu(y)\right\}^{1 / r^{\prime}} \\
\quad \times\left\{\frac{1}{\mu\left(\frac{3}{2} Q\right)} \int_{Q}\left|I_{\alpha} f(y)\right|^{r} d \mu(y)\right\}^{1 / r} \\
\leq C\left\|b_{1}\right\|_{*}\left\|b_{2}\right\|_{*} M_{r,(3 / 2)}\left(I_{\alpha} f\right)(x),
\end{aligned}
$$


and

$$
\begin{aligned}
\mathrm{I}_{2} \leq\left[\frac{1}{\mu\left(\frac{3}{2} Q\right)} \int_{Q}\left|b_{1}(y)-b_{Q}^{1}\right|^{r^{\prime}} d \mu(y)\right]^{1 / r^{\prime}} \\
\quad \times\left[\frac{1}{\mu\left(\frac{3}{2} Q\right)} \int_{Q}\left|I_{\alpha ; b_{2}} f(y)\right|^{r} d \mu(y)\right]^{1 / r} \\
\leq C\left\|b_{1}\right\|_{*} M_{r,(3 / 2)}\left(I_{\alpha ; b_{2}} f\right)(x) .
\end{aligned}
$$

An estimate similar to that for $\mathrm{I}_{2}$ tells us that

$$
\mathrm{I}_{3} \leq C\left\|b_{2}\right\|_{*} M_{r,(3 / 2)}\left(I_{\alpha ; b_{1}} f\right)(x) .
$$

To estimate $\mathrm{I}_{4}$, let $f_{1}=f \chi_{\frac{4}{3} Q}$ and $f_{2}=f \chi_{\mathbb{R}^{d} \backslash \frac{4}{3} Q}$. Decompose $\mathrm{I}_{4}$ into

$$
\begin{aligned}
\mathrm{I}_{4} \leq & \frac{1}{\mu\left(\frac{3}{2} Q\right)} \int_{Q}\left|I_{\alpha}\left[\left(b_{1}-b_{Q}^{1}\right)\left(b_{2}-b_{Q}^{2}\right) f_{1}\right](y)\right| d \mu(y) \\
& +\frac{1}{\mu\left(\frac{3}{2} Q\right)} \int_{Q}\left|I_{\alpha}\left[\left(b_{1}-b_{Q}^{1}\right)\left(b_{2}-b_{Q}^{2}\right) f_{2}\right](y)-h_{Q}\right| d \mu(y) \\
= & \mathrm{E}+\mathrm{F} .
\end{aligned}
$$

Let $s=\sqrt{r}$ and $1 / \nu=1 / s-\alpha / n$. From the Hölder inequality, (2.1) and Lemma 2.1, it follows that

$$
\begin{aligned}
\mathrm{E} & \leq C \frac{\mu(Q)^{1-1 / \nu}}{\mu\left(\frac{3}{2} Q\right)}\left\|I_{\alpha}\left[\left(b_{1}-b_{Q}^{1}\right)\left(b_{2}-b_{Q}^{2}\right) f_{1}\right]\right\|_{L^{\nu}(\mu)} \\
& \leq C \frac{\mu(Q)^{1-1 / \nu}}{\mu\left(\frac{3}{2} Q\right)}\left\|\left(b_{1}-b_{Q}^{1}\right)\left(b_{2}-b_{Q}^{2}\right) f_{1}\right\|_{L^{s}(\mu)} \\
& \leq C\left\|b_{1}\right\|_{*}\left\|b_{2}\right\|_{*} M_{r,(9 / 8)}^{(\alpha)} f(x) .
\end{aligned}
$$


To estimate $\mathrm{F}$, by the Hölder inequality, we first have that for $y_{1}, y_{2} \in Q$,

$(2.10)$

$$
\begin{aligned}
& \left|I_{\alpha}\left[\left(b_{1}-b_{Q}^{1}\right)\left(b_{2}-b_{Q}^{2}\right) f_{2}\right]\left(y_{1}\right)-I_{\alpha}\left[\left(b_{1}-b_{Q}^{1}\right)\left(b_{2}-b_{Q}^{2}\right) f_{2}\right]\left(y_{2}\right)\right| \\
& \leq C \int_{\mathbb{R}^{d} \backslash \frac{4}{3} Q} \frac{\left|y_{1}-y_{2}\right|}{\left|z-y_{1}\right|^{n+1-\alpha}}\left|\left(b_{1}(z)-b_{Q}^{1}\right)\left(b_{2}(z)-b_{Q}^{2}\right)\right||f(z)| d \mu(z) \\
& \leq C \sum_{k=1}^{\infty} \int_{2^{k} \frac{4}{3} Q \backslash 2^{k-1} \frac{4}{3} Q} \frac{l(Q)}{\left|z-y_{1}\right|^{n+1-\alpha}} \\
& \times\left|\left(b_{1}(z)-b_{2^{k} \frac{4}{3} Q}^{1}\right)\left(b_{2}(z)-b_{2^{k \frac{4}{3} Q}}^{2}\right)\right||f(z)| d \mu(z) \\
& +C \sum_{k=1}^{\infty}\left|b_{Q}^{1}-b_{2^{k} \frac{4}{3} Q}^{1}\right| \int_{2^{k \frac{4}{3} Q \backslash 2^{k-1} \frac{4}{3} Q}} \frac{l(Q)}{\left|z-y_{1}\right|^{n+1-\alpha}} \\
& \times\left|b_{2}(z)-b_{2^{k} \frac{4}{3} Q}^{2}\right| f(z) \mid d \mu(z) \\
& +C \sum_{k=1}^{\infty}\left|b_{Q}^{2}-b_{2^{k} \frac{4}{3} Q}^{2}\right| \int_{2^{k \frac{4}{3} Q \backslash 2^{k-1} \frac{4}{3} Q}} \frac{l(Q)}{\left|z-y_{1}\right|^{n+1-\alpha}} \\
& \times\left|b_{1}(z)-b_{2^{k} \frac{4}{3} Q}^{1}\right| f(z) \mid d \mu(z) \\
& +C \sum_{k=1}^{\infty}\left|\left(b_{Q}^{1}-b_{2^{k} \frac{4}{3} Q}^{1}\right)\left(b_{Q}^{2}-b_{2^{k} \frac{4}{3} Q}^{2}\right)\right| \int_{2^{k \frac{4}{3} Q \backslash 2^{k-1 \frac{4}{3} Q}} \mid} \frac{l(Q)}{\left|z-y_{1}\right|^{n+1-\alpha}}|f(z)| d \mu(z) \\
& \leq C \sum_{k=1}^{\infty} k^{2} 2^{-k}\left\|b_{1}\right\|_{*}\left\|b_{2}\right\|_{*}\left[\frac{1}{\mu\left(2^{k} \frac{3}{2} Q\right)^{1-\alpha r / n}} \int_{2^{k} \frac{4}{3} Q}|f(z)|^{r} d \mu(z)\right]^{1 / r} \\
& \leq C\left\|b_{1}\right\|_{*}\left\|b_{2}\right\|_{*} M_{r,(9 / 8)}^{(\alpha)} f(x),
\end{aligned}
$$

where we used the estimate

$$
\left|b_{j}-b_{2^{k \frac{4}{3} Q}}^{j}\right| \leq C k\left\|b_{j}\right\|_{* *} \leq C k\left\|b_{j}\right\|_{*}
$$

for $j=1,2$. From (2.10) and the choice of $h_{Q}$, it follows that

$$
\left|I_{\alpha}\left[\left(b_{1}-b_{Q}^{1}\right)\left(b_{2}-b_{Q}^{2}\right) f_{2}\right]\left(y_{1}\right)-h_{Q}\right| \leq C\left\|b_{1}\right\|_{*}\left\|b_{2}\right\|_{*} M_{r,(9 / 8)}^{(\alpha)} f(x) .
$$


Therefore,

$$
\mathrm{F} \leq C\left\|b_{1}\right\|_{*}\left\|b_{2}\right\|_{*} M_{r,(9 / 8)}^{(\alpha)} f(x) .
$$

The estimates (2.9) and (2.11) indicate

$$
\mathrm{I}_{4} \leq C\left\|b_{1}\right\|_{*}\left\|b_{2}\right\|_{*} M_{r,(9 / 8)}^{(\alpha)} f(x)
$$

Combining (2.6), (2.7), (2.8) and (2.12) yields (2.4).

We now check (2.5) for chosen $\left\{h_{Q}\right\}_{Q}$ as above. Consider two cubes $Q \subset R$ with $x \in Q$ and a doubling cube $R$. Denote $N_{Q, R}+1$ simply by $N$. Write

$$
\begin{aligned}
\left|h_{Q}-h_{R}\right|= & \mid m_{Q}\left(I_{\alpha}\left[\left(b_{1}-b_{Q}^{1}\right)\left(b_{2}-b_{Q}^{2}\right) f \chi_{\mathbb{R}^{d} \backslash \frac{4}{3} Q}\right]\right) \\
& -m_{R}\left(I_{\alpha}\left[\left(b_{1}-b_{R}^{1}\right)\left(b_{2}-b_{R}^{2}\right) f \chi_{\mathbb{R}^{d} \backslash \frac{4}{3} R}\right]\right) \mid \\
\leq & \mid m_{R}\left(I_{\alpha}\left[\left(b_{1}-b_{Q}^{1}\right)\left(b_{2}-b_{Q}^{2}\right) f \chi_{\mathbb{R}^{d} \backslash 2^{N} Q}\right]\right) \\
& -m_{Q}\left(I _ { \alpha } \left[\left(b_{1}-b_{Q}^{1}\right)\left(b_{2}-b_{Q}^{2}\right) f \chi_{\mathbb{R}^{d} \backslash 2^{N} Q}\left(I_{\alpha}\left[\left(b_{1}-b_{R}^{1}\right)\left(b_{2}-b_{R}^{2}\right) f \chi_{\mathbb{R}^{d} \backslash 2^{N} Q}\right]\right)\right.\right. \\
& -m_{R}\left(I_{\alpha}\left[\left(b_{1}-b_{Q}^{1}\right)\left(b_{2}-b_{Q}^{2}\right) f \chi_{\mathbb{R}^{d} \backslash 2^{N} Q}\right]\right) \mid \\
& +\left|m_{Q}\left(I_{\alpha}\left[\left(b_{1}-b_{Q}^{1}\right)\left(b_{2}-b_{Q}^{2}\right) f \chi_{2^{N} Q \backslash \frac{4}{3} Q}\right]\right)\right| \\
& +\left|m_{R}\left(I_{\alpha}\left[\left(b_{1}-b_{R}^{1}\right)\left(b_{2}-b_{R}^{2}\right) f \chi_{2^{N} Q \backslash \frac{4}{3} R}\right]\right)\right| \\
= & \mathrm{L}_{1}+\mathrm{L}_{2}+\mathrm{L}_{3}+\mathrm{L}_{4} .
\end{aligned}
$$

Similar to the estimate for F, we easily obtain

$$
\mathrm{L}_{1} \leq C K_{Q, R}^{2}\left\|b_{1}\right\|_{*}\left\|b_{2}\right\|_{*} M_{r,(9 / 8)}^{(\alpha)} f(x)
$$


We estimate $\mathrm{F}_{2}$ by decomposing it into

$$
\begin{aligned}
\mathrm{L}_{2} \leq & \mid m_{R}\left(I_{\alpha}\left[\left(b_{1}-b_{R}^{1}\right)\left(b_{2}-b_{R}^{2}\right) f \chi_{\mathbb{R}^{d} \backslash 2^{N} Q}\right]\right) \\
& -m_{R}\left(I_{\alpha}\left[\left(b_{1}-b_{R}^{1}+b_{R}^{1}-b_{Q}^{1}\right)\left(b_{2}-b_{R}^{2}+b_{R}^{2}-b_{Q}^{2}\right) f \chi_{\mathbb{R}^{d} \backslash 2^{N} Q}\right]\right) \mid \\
\leq & C K_{Q, R}\left\|b_{2}\right\|_{*}\left|m_{R}\left(I_{\alpha}\left[\left(b_{1}-b_{R}^{1}\right) f \chi_{\mathbb{R}^{d} \backslash 2^{N} Q}\right]\right)\right| \\
& +C K_{Q, R}\left\|b_{1}\right\|_{*}\left|m_{R}\left(I_{\alpha}\left[\left(b_{2}-b_{R}^{2}\right) f \chi_{\mathbb{R}^{d} \backslash 2^{N} Q}\right]\right)\right| \\
& +C K_{Q, R}^{2}\left\|b_{1}\right\|_{*}\left\|b_{2}\right\|_{*}\left|m_{R}\left[I_{\alpha}\left(f \chi_{\mathbb{R}^{d} \backslash 2^{N} Q}\right)\right]\right| \\
= & \mathrm{M}_{1}+\mathrm{M}_{2}+\mathrm{M}_{3} .
\end{aligned}
$$

From the fact that $R$ is a doubling cube, it is easy to deduce that

$$
\mathrm{M}_{3} \leq C K_{Q, R}^{2}\left\|b_{1}\right\|_{*}\left\|b_{2}\right\|_{*} M_{r,(3 / 2)}\left[I_{\alpha}(|f|)\right](x) .
$$

We further estimate $M_{1}$ by writing

$$
\begin{aligned}
I_{\alpha}\left[\left(b_{1}-b_{R}^{1}\right) f \chi_{\mathbb{R}^{d} \backslash 2^{N} Q}\right](y)= & I_{\alpha}\left[\left(b_{1}-b_{R}^{1}\right) f\right](y)-I_{\alpha}\left[\left(b_{1}-b_{R}^{1}\right) f \chi_{2^{N} Q}\right](y) \\
= & \left(b_{1}(y)-b_{R}^{1}\right) I_{\alpha}(f)(y)-I_{\alpha ; b_{1}}(f)(y) \\
& -I_{\alpha}\left[\left(b_{1}-b_{R}^{1}\right) f \chi_{\frac{4}{3} R}\right](y) \\
& -I_{\alpha}\left[\left(b_{1}-b_{R}^{1}\right) f \chi_{2^{N} Q \backslash \frac{4}{3} R}\right](y) .
\end{aligned}
$$

From the fact that $R$ is a doubling cube, it follows that

$$
\frac{1}{\mu(R)} \int_{R}\left|b_{1}(y)-b_{R}^{1}\right|\left|I_{\alpha}(f)(y)\right| d \mu(y) \leq C\left\|b_{1}\right\|_{*} M_{r,(3 / 2)} I_{\alpha}(f)(x)
$$

and

$$
\frac{1}{\mu(R)} \int_{R}\left|I_{\alpha ; b_{1}}(f)(y)\right| d \mu(y) \leq C M_{r,(3 / 2)}\left(I_{\alpha ; b_{1}} f\right)(x) .
$$

An estimate similar to that for $\mathrm{E}$ and the fact that $R$ is a doubling cube tell us that

$$
\frac{1}{\mu(R)} \int_{R}\left|I_{\alpha}\left[\left(b_{1}-b_{R}^{1}\right) f \chi_{\frac{4}{3} R}\right](y)\right| d \mu(y) \leq C\left\|b_{1}\right\|_{*} M_{r,(9 / 8)}^{(\alpha)} f(x) .
$$


Noting that $l\left(2^{N} Q\right) \sim l(R)$ and $2^{N} Q \supset R$, by the Hölder inequality and (2.1), for $y \in R$, we have

$$
\begin{aligned}
& \left|I_{\alpha}\left[\left(b_{1}-b_{R}^{1}\right) f \chi_{2^{N} Q \backslash \frac{4}{3} R}\right](y)\right|=\left|\int_{2^{N} Q \backslash \frac{4}{3} R} \frac{b_{1}(z)-b_{R}^{1}}{|y-z|^{n-\alpha}} f(z) d \mu(z)\right| \\
& \leq \frac{C}{l(R)^{n-\alpha}} \int_{2^{N} Q}\left|b_{1}(z)-b_{R}^{1}\right||f(z)| d \mu(z) \\
& \leq \frac{C}{\mu\left(2^{N+1} Q\right)^{1-\alpha / n}}\left\{\left[\int_{2^{N} Q}\left|b_{1}(z)-b_{2^{N} Q}^{1}\right|^{r^{\prime}} d \mu(z)\right]^{1 / r^{\prime}}\right. \\
& \left.\quad \times\left[\int_{2^{N} Q}|f(z)|^{r} d \mu(z)\right]^{1 / r}+\left|b_{2^{N} Q}^{1}-b_{R}^{1}\right| \mu\left(2^{N} Q\right)^{1 / r^{\prime}}\right\} \\
& \leq C\left\|b_{1}\right\|_{*}\left[\frac{1}{\mu\left(2^{N+1} Q\right)^{1-\alpha r / n}} \int_{2^{N} Q}|f(z)|^{r} d \mu(z)\right]^{1 / r} \\
& \leq C\left\|b_{1}\right\|_{*} M_{r,(9 / 8)}^{(\alpha)} f(x) .
\end{aligned}
$$

Thus

$$
\frac{1}{\mu(R)} \int_{R}\left|I_{\alpha}\left[\left(b_{1}-b_{R}^{1}\right) f \chi_{2^{N} Q \backslash \frac{4}{3} R}\right](y)\right| d \mu(y) \leq C\left\|b_{1}\right\|_{*} M_{r,(9 / 8)}^{(\alpha)} f(x) .
$$

All the estimates above lead to

$$
\begin{aligned}
\mathrm{M}_{1} \leq & C K_{Q, R}\left\|b_{1}\right\|_{*}\left\|b_{2}\right\|_{*}\left\{M_{r,(3 / 2)}\left(I_{\alpha} f\right)(x)+M_{r,(9 / 8)}^{(\alpha)}(f)(x)\right\} \\
& +C K_{Q, R}\left\|b_{2}\right\|_{*} M_{r,(3 / 2)}\left(I_{\alpha ; b_{1}} f\right)(x) .
\end{aligned}
$$

Similarly, we can prove

$$
\begin{aligned}
\mathrm{M}_{2} \leq & C K_{Q, R}\left\|b_{1}\right\|_{*}\left\|b_{2}\right\|_{*}\left\{M_{r,(3 / 2)}\left(I_{\alpha} f\right)(x)+M_{r,(9 / 8)}^{(\alpha)}(f)(x)\right\} \\
& +C K_{Q, R}\left\|b_{1}\right\|_{*} M_{r,(3 / 2)}\left(I_{\alpha ; b_{2}} f\right)(x) .
\end{aligned}
$$


Combining the estimates (2.14), (2.15) and (2.16) leads to

$$
\begin{aligned}
\mathrm{L}_{2} \leq & C\left\|b_{1}\right\|_{*}\left\|b_{2}\right\|_{*} K_{Q, R}^{2}\left\{M_{r,(9 / 8)}^{(\alpha)} f(x)+M_{r,(3 / 2)}\left[I_{\alpha}(|f|)\right](x)\right\} \\
& +C\left\|b_{1}\right\|_{*} K_{Q, R} M_{r,(3 / 2)}\left(I_{\alpha ; b_{2}} f\right)(x) \\
& +C\left\|b_{2}\right\|_{*} K_{Q, R} M_{r,(3 / 2)}\left(I_{\alpha ; b_{1}} f\right)(x) .
\end{aligned}
$$

Let us now estimate $\mathrm{L}_{3}$ by first decomposing

$$
\begin{aligned}
\mid I_{\alpha}[ & \left.\left(b_{1}-b_{Q}^{1}\right)\left(b_{2}-b_{Q}^{2}\right) f \chi_{2^{N} Q \backslash \frac{4}{3} Q}\right](y) \mid \\
\leq & \left|I_{\alpha}\left[\left(b_{1}-b_{Q}^{1}\right)\left(b_{2}-b_{Q}^{2}\right) f \chi_{2 Q \backslash \frac{4}{3} Q}\right](y)\right| \\
& +\left|I_{\alpha}\left[\left(b_{1}-b_{Q}^{1}\right)\left(b_{2}-b_{Q}^{2}\right) f \chi_{2^{N} Q \backslash 2 Q}\right](y)\right| \\
\leq & C\left\|b_{1}\right\|_{*}\left\|b_{2}\right\|_{*} M_{r,(9 / 8)}^{(\alpha)} f(x) \\
& +C \sum_{k=1}^{N-1} \frac{1}{l\left(2^{k} Q\right)^{n-\alpha}} \int_{2^{k+1} Q \backslash 2^{k} Q}\left|\left(b_{1}(z)-b_{Q}^{1}\right)\left(b_{2}(z)-b_{Q}^{2}\right)\right||f(z)| d \mu(z) \\
\leq & C\left\|b_{1}\right\|_{*}\left\|b_{2}\right\|_{*} M_{r,(9 / 8)}^{(\alpha)} f(x) \\
& +C K_{Q, R}^{2}\left\|b_{1}\right\|_{*}\left\|b_{2}\right\|_{*} \\
& \times \sum_{k=1}^{N-1} \frac{\mu\left(2^{k+2} Q\right)^{1-\alpha / n}}{l\left(2^{k} Q\right)^{n-\alpha}}\left[\frac{1}{\mu\left(2^{k+2} Q\right)^{1-\alpha r / n}} \int_{2^{k+1} Q}|f(z)|^{r} d \mu(z)\right]^{1 / r} \\
\leq & C K_{Q, R}^{2} K_{Q, R}^{(\alpha)}\left\|b_{1}\right\|_{*}\left\|b_{2}\right\|_{*} M_{r,(9 / 8)}^{(\alpha)} f(x),
\end{aligned}
$$

where we used the Hölder inequality and (2.1). Taking the mean over $Q$, we then obtain

$$
\mathrm{L}_{3} \leq C K_{Q, R}^{2} K_{Q, R}^{(\alpha)}\left\|b_{1}\right\|_{*}\left\|b_{2}\right\|_{*} M_{r,(9 / 8)}^{(\alpha)} f(x) .
$$

Similarly, we have

$$
\mathrm{L}_{4} \leq C\left\|b_{1}\right\|_{*}\left\|b_{2}\right\|_{*} M_{r,(9 / 8)}^{(\alpha)} f(x) .
$$

Combining the estimates (2.13), (2.17), (2.18) and (2.19) yields the estimate (2.5) and this finishes the proof of Theorem 1.1. 


\section{Proof of Theorem 1.2}

We begin with some preliminary lemmas.

Lemma 3.1 ([20]). Let $b \in \operatorname{RBMO}(\mu)$. There exist two positive constants $C_{1}$ and $C_{2}$ such that for any cube $Q$ and $\lambda>0$.

$$
\mu\left(\left\{x \in Q:\left|b(x)-m_{\widetilde{Q}}(b)\right|>\lambda\right\}\right) \leq C_{1} \mu(2 Q) \exp \left(\frac{-C_{2} \lambda}{\|b\|_{\operatorname{RBMO}(\mu)}}\right) .
$$

The following lemma on the Calderón-Zygmund decomposition in non-homogeneous spaces can be found in [22], [19].

Lemma 3.2. Assume that $\mu$ satisfies (1.1). For any $f \in L^{1}(\mu)$ and any $\lambda>0$ (with $\lambda>2^{d+1}\|f\|_{L^{1}(\mu)} /\|\mu\|$ if $\left.\|\mu\|<\infty\right)$, we have

(a) There exists a family of almost disjoint cubes $\left\{Q_{j}\right\}_{j}$ (that is, $\sum_{j} \chi_{Q_{j}}(x) \leq C$ ) such that

$$
\frac{\lambda}{2^{d+1}}<\frac{1}{\mu\left(2 Q_{j}\right)} \int_{Q_{j}}|f(x)| d \mu(x)
$$

and

$$
\frac{1}{\mu\left(2 \eta Q_{j}\right)} \int_{\eta Q_{j}}|f(x)| d \mu(x) \leq \frac{\lambda}{2^{d+1}}
$$

for all $\eta>2$.

(b) $|f(x)| \leq \lambda \mu$-a.e. on $\mathbb{R}^{d} \backslash \cup_{j} Q_{j}$.

(c) For each fixed $j$, let $R_{j}$ be a $\left(6,6^{n+1}\right)$-doubling cube concentric with $Q_{j}$, with $l\left(R_{j}\right)>4 l\left(Q_{j}\right)$ and set $w_{j}=\chi_{Q_{j}} / \sum_{k} \chi_{Q_{k}}$. Then there exists a family of functions $\phi_{j}$ with $\operatorname{supp} \phi_{j} \subset R_{j}$ and with constant sign satisfying

$$
\begin{gathered}
\int_{\mathbb{R}^{d}} \phi_{j}(x) d \mu(x)=\int_{Q_{j}} f(x) w_{j}(x) d \mu(x), \\
\left\|\phi_{j}\right\|_{L^{\infty}(\mu)} \mu\left(R_{j}\right) \leq C \int_{Q_{j}}|f(x)| d \mu(x)
\end{gathered}
$$

and

$$
\sum_{j}\left|\phi_{j}(x)\right| \leq C \lambda
$$

Proof of Theorem 1.2: To prove the theorem, without loss of generality, we may assume that $\|f\|_{L^{1}(\mu)}=1$ and $\left\|b_{j}\right\|_{\mathrm{Osc}_{\exp L^{r_{j}}}}(\mu)=1$ for 
$j=1, \ldots, m$. In fact, let

$$
\widetilde{b}_{j}=\frac{b_{j}}{\left\|b_{j}\right\|_{\mathrm{Osc}_{\exp L^{r_{j}}(\mu)}}}
$$

for $j=1, \ldots, m$. The homogeneity tells us that

(3.1) $\mu\left(\left\{x \in \mathbb{R}^{d}:\left|I_{\alpha ; b_{1}, \ldots, b_{m}} f(x)\right|>\lambda\right\}\right)$

$$
\begin{aligned}
=\mu\left(\left\{x \in \mathbb{R}^{d}\right.\right. & :\left|I_{\alpha ; \widetilde{b}_{1}, \ldots, \widetilde{b}_{m}}\left[\frac{f(x)}{\|f\|_{L^{1}(\mu)}}\right]\right| \\
& \left.\left.>\frac{\lambda}{\|f\|_{L^{1}(\mu)} \prod_{j=1}^{m}\left\|b_{j}\right\|_{\operatorname{Osc}_{\exp ^{r_{j}}(\mu)}}}\right\}\right) .
\end{aligned}
$$

Noting that

$$
\left\|\frac{f}{\|f\|_{L^{1}(\mu)}}\right\|_{L^{1}(\mu)}=1
$$

and

$$
\left\|\widetilde{b}_{j}\right\|_{\operatorname{Osc}_{\exp L_{j} r_{j}(\mu)}}=1
$$

for $j=1, \ldots, m$, if the theorem is true when $\|f\|_{L^{1}(\mu)}=1$ and

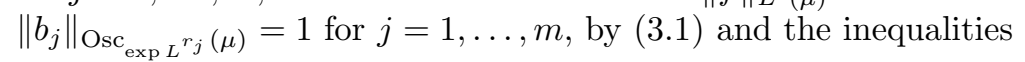

$$
\Phi_{s}\left(t_{1} t_{2}\right) \leq C \Phi_{s}\left(t_{1}\right) \Phi_{s}\left(t_{2}\right)
$$

for any $s>0, t_{1}, t_{2} \geq 0$ and

$$
\Phi_{1 / r_{\sigma}}\left[\Phi_{1 / r_{\sigma^{\prime}}}(t)\right] \leq C \Phi_{1 / r}(t)
$$

for $t \geq 0$, we easily deduce that the theorem still holds for any bounded $f$ with compact support and any $b_{j} \in \mathrm{Osc}_{\exp } L^{r_{j}}(\mu)$ for $j=1, \ldots, m$.

In what follows, we prove the theorem by two steps.

Step I: In this step, we prove Theorem 1.2 for $m=1$.

For any fixed bounded and compact supported function $f$ and any fixed

$$
\lambda>2^{d+1}\|f\|_{L^{1}(\mu)} /\|\mu\|,
$$

applying the Calderón-Zygmund decomposition (see Lemma 3.2) to $f$ at level $\lambda^{q_{0}}$, we obtain a sequence of cubes $\left\{Q_{j}\right\}_{j}$ with bounded overlaps, that is,

$$
\sum_{j} \chi_{Q_{j}}(x) \leq C<\infty
$$


such that

(I)

$$
\frac{\lambda^{q_{0}}}{2^{d+1}}<\frac{1}{\mu\left(2 Q_{j}\right)} \int_{Q_{j}}|f(x)| d \mu(x)
$$

and

$$
\frac{1}{\mu\left(2 \eta Q_{j}\right)} \int_{\eta Q_{j}}|f(x)| d \mu(x) \leq \frac{\lambda^{q_{0}}}{2^{d+1}}
$$

for all $\eta>2$.

(II) $|f(x)| \leq \lambda^{q_{0}} \mu$-a.e. on $\mathbb{R}^{d} \backslash \cup_{j} Q_{j}$.

(III) For each fixed $j$, let $R_{j}$ be the smallest $\left(6,6^{n+1}\right)$-doubling cube of the form $6^{k} Q_{j}, k \geq 1$. Set $w_{j}=\chi_{Q_{j}} / \sum_{k} \chi_{Q_{k}}$. Then there are a function $\phi_{j}$ with supp $\phi_{j} \subset R_{j}$ and a constant $C>0$ satisfying

$$
\begin{gathered}
\int_{\mathbb{R}^{d}} \phi_{j}(x) d \mu(x)=\int_{Q_{j}} f(x) w_{j}(x) d \mu(x), \\
\left\|\phi_{j}\right\|_{L^{\infty}(\mu)} \mu\left(R_{j}\right) \leq C \int_{Q_{j}}|f(x)| d \mu(x)
\end{gathered}
$$

and

$$
\sum_{j}\left|\phi_{j}(x)\right| \leq C \lambda^{q_{0}}
$$

It is easy to see that the conclusion of the theorem still holds if

$$
\lambda \leq 2^{d+1}\|f\|_{L^{1}(\mu)} /\|\mu\|
$$

when $\|\mu\|<\infty$.

Decompose $f$ into $f=g+h$, where

$$
g(x)=f(x) \chi_{\mathbb{R}^{d} \backslash \cup_{j} Q_{j}}(x)+\sum_{j} \phi_{j}(x),
$$

and

$$
h(x)=f(x)-g(x)=\sum_{j}\left[w_{j}(x) f(x)-\phi_{j}(x)\right]=\sum_{j} h_{j}(x) .
$$


Let $1<p_{1}<n / \alpha$ and $1 / q_{1}=1 / p_{1}-\alpha / n$. Recall that

$$
\|g\|_{L^{1}(\mu)} \leq C\|f\|_{L^{1}(\mu)} \leq C
$$

and $I_{\alpha ; b_{1}}$ is bounded from $L^{p_{1}}(\mu)$ to $L^{q_{1}}(\mu)$ by Theorem 1.1 (see also [2]). This via the fact that $\|g\|_{L^{\infty}(\mu)} \leq C \lambda^{q_{0}}$ gives

$$
\begin{aligned}
\mu\left(\left\{x \in \mathbb{R}^{d}:\left|I_{\alpha ; b_{1}} g(x)\right|>\lambda\right\}\right) & \leq C \lambda^{-q_{1}} \int_{\mathbb{R}^{d}}\left|I_{\alpha ; b_{1}} g(y)\right|^{q_{1}} d \mu(y) \\
& \leq C \lambda^{-q_{1}}\|g\|_{L^{p_{1}}(\mu)}^{q_{1}} \\
& \leq C \lambda^{-q_{1}} \lambda^{q_{0}\left(p_{1}-1\right) q_{1} / p_{1}}\|f\|_{L^{1}(\mu)}^{q_{1} / p_{1}} \\
& \leq C \lambda^{-q_{0}} .
\end{aligned}
$$

Noting that $r=r_{1}$ when $m=1$ and

$$
\mu\left(\bigcup_{j} 2 Q_{j}\right) \leq C \lambda^{-q_{0}} \int_{\mathbb{R}^{d}}|f(y)| d \mu(y) \leq C \lambda^{-q_{0}}
$$

therefore, the proof of the theorem can be reduced to proving that

$$
\begin{aligned}
& \mu\left(\left\{x \in \mathbb{R}^{d} \backslash \bigcup_{j} 2 Q_{j}:\left|I_{\alpha ; b_{1}} h(x)\right|>\lambda\right\}\right) \\
& \quad \leq C\left[\left\|\Phi_{1 / r}\left(\lambda^{-1}|f|\right)\right\|_{L^{1}(\mu)}+\Phi_{1 / r}\left(\lambda^{-1}\|f\|_{L^{1}(\mu)}\right)\right]^{q_{0}}
\end{aligned}
$$

For each fixed $j$, set $b_{1}^{(j)}(x)=b_{1}(x)-m_{\widetilde{Q_{j}}}\left(b_{1}\right)$ and write

$$
I_{\alpha ; b_{1}} h(x)=\sum_{j} b_{1}^{(j)}(x) I_{\alpha} h_{j}(x)+I_{\alpha}\left(b_{1}^{(j)} h_{j}\right)(x)=\mathrm{I}(x)+\mathrm{I} I(x) .
$$


The weak type $\left(1, q_{0}\right)$ boundedness of $I_{\alpha}$ (see Proposition 6.2 in $[\mathbf{6}]$ ) tells us that

$$
\begin{aligned}
\mu(\{x \in & \left.\left.\mathbb{R}^{d}:|\mathrm{I} I(x)|>\lambda\right\}\right) \\
\leq & C \lambda^{-q_{0}}\left[\sum_{j} \int_{\mathbb{R}^{d}}\left|b_{1}^{(j)}(y) h_{j}(y)\right| d \mu(y)\right]^{q_{0}} \\
\leq & C \lambda^{-q_{0}}\left[\sum_{j} \int_{Q_{j}}\left|b_{1}(y)-m_{\widetilde{Q_{j}}}\left(b_{1}\right)\right||f(y)| d \mu(y)\right]^{q_{0}} \\
& +C \lambda^{-q_{0}}\left[\sum_{j}\left\|\phi_{j}\right\|_{L^{\infty}(\mu)} \int_{R_{j}}\left|b_{1}(y)-m_{\widetilde{Q_{j}}}\left(b_{1}\right)\right| d \mu(y)\right]^{q_{0}} \\
= & \mathrm{U}+\mathrm{V} .
\end{aligned}
$$

It is obvious that $R_{j}$ is also $\left(2, \beta_{d}\right)$-doubling and $R_{j}=\widetilde{R_{j}}$. Thus, by Lemma 2.8 in $[\mathbf{2 0}]$,

$$
\begin{aligned}
\int_{R_{j}}\left|b_{1}(y)-m_{\widetilde{Q_{j}}}\left(b_{1}\right)\right| d \mu(y) \leq \int_{R_{j}}\left|b_{1}(y)-m_{R_{j}}\left(b_{1}\right)\right| d \mu(y) \\
+\mu\left(R_{j}\right)\left[\left|m_{\widetilde{6 Q_{j}}}\left(b_{1}\right)-m_{R_{j}}\left(b_{1}\right)\right|\right. \\
\left.+\left|m_{\widetilde{6 Q_{j}}}\left(b_{1}\right)-m_{\widetilde{Q_{j}}}\left(b_{1}\right)\right|\right] \\
\leq C \mu\left(2 R_{j}\right)+C \mu\left(R_{j}\right)\left(K_{6 Q_{j}, R_{j}}+K_{6 Q_{j}, Q_{j}}\right) .
\end{aligned}
$$

A trivial computation shows that $K_{6 Q_{j}, R_{j}} \leq C$ (see also Lemma 2.1 (3) in [20]). This together with the estimate $\mu\left(2 R_{j}\right) \leq \mu\left(6 R_{j}\right) \leq 6^{n+1} \mu\left(R_{j}\right)$ in turn implies that

$$
\mathrm{V} \leq C \lambda^{-q_{0}}\left[\sum_{j}\left\|\phi_{j}\right\|_{L^{\infty}(\mu)} \mu\left(R_{j}\right)\right]^{q_{0}} \leq C \lambda^{-q_{0}}\left[\int_{\mathbb{R}^{d}}|f(y)| d \mu(y)\right]^{q_{0}}
$$

which is a desired estimate for $\mathrm{V}$.

On the other hand, by the generalized Hölder inequality (1.3) and Lemma 3.1, we have

$$
\begin{aligned}
\mathrm{U} & \leq C \lambda^{-q_{0}}\left\{\sum_{j} \mu\left(2 Q_{j}\right)\|f\|_{L \log ^{1 / r} L, Q_{j}, \mu / \mu\left(2 Q_{j}\right)}\left\|b_{1}^{(j)}\right\|_{\exp L^{r}, Q_{j}, \mu / \mu\left(2 Q_{j}\right)}\right\}^{q_{0}} \\
& \leq C \lambda^{-q_{0}}\left\{\sum_{j} \mu\left(2 Q_{j}\right)\|f\|_{L \log ^{1 / r} L, Q_{j}, \mu / \mu\left(2 Q_{j}\right)}\right\}^{q_{0}}
\end{aligned}
$$


From the fact that

$$
\begin{aligned}
\|f\|_{L \log ^{1 / r}} L, Q_{j}, \mu / \mu\left(2 Q_{j}\right) \\
\quad \leq \inf \left\{t+\frac{t}{\mu\left(2 Q_{j}\right)} \int_{Q_{j}} \frac{|f(y)|}{t} \log ^{1 / r}\left(2+\frac{|f(y)|}{t}\right) d \mu(y)\right\} \\
\quad \leq \lambda^{q_{0}}+\frac{1}{\mu\left(2 Q_{j}\right)} \int_{Q_{j}}|f(y)| \log ^{1 / r}\left(2+\frac{|f(y)|}{\lambda^{q_{0}}}\right) d \mu(y)
\end{aligned}
$$

(see $[\mathbf{1 6}]$ and the related references there) and the inequality

$$
\log ^{1 / r}\left(2+t_{1} t_{2}\right) \leq C\left[\log ^{1 / r}\left(2+t_{1}\right)+\log ^{1 / r}\left(2+t_{1}\right)\right]
$$

for $t_{1}, t_{2} \geq 0$, it follows that

$$
\begin{aligned}
\mathrm{U} \leq C \lambda^{-q_{0}}\left\{\sum_{j} \mu\left(2 Q_{j}\right) \lambda^{q_{0}}\right. & +\sum_{j} \int_{Q_{j}}|f(y)| \log ^{1 / r}\left(2+\frac{|f(y)|}{\lambda}\right) d \mu(y) \\
& \left.+\sum_{j} \int_{Q_{j}}|f(y)| d \mu(y) \log ^{1 / r}\left(2+\frac{1}{\lambda}\right)\right\}^{q_{0}} \\
\leq C\left\{\frac{1}{\lambda}\|f\|_{L^{1}(\mu)}+\lambda^{-1} \log ^{1 / r}\left(2+\frac{1}{\lambda}\right)\|f\|_{L^{1}(\mu)}\right. & \left.+\int_{\mathbb{R}^{d}} \frac{|f(y)|}{\lambda} \log ^{1 / r}\left(2+\frac{|f(y)|}{\lambda}\right) d \mu(y)\right\}^{q_{0}} \\
\leq C\left\{\Phi_{1 / r}\left(\lambda^{-1}\|f\|_{L^{1}(\mu)}\right)\right. & \left.+\left\|\Phi_{1 / r}\left(\lambda^{-1}|f|\right)\right\|_{L^{1}(\mu)}\right\}^{q_{0}},
\end{aligned}
$$

which is a desired estimate for $\mathrm{U}$.

Combining (3.5) and (3.6), we obtain that

$$
\begin{aligned}
\mu\left(\left\{x \in \mathbb{R}^{d}:|\mathrm{I} I(x)|>\lambda\right\}\right) \\
\quad \leq C\left\{\Phi_{1 / r}\left(\lambda^{-1}\|f\|_{L^{1}(\mu)}\right)+\left\|\Phi_{1 / r}\left(\lambda^{-1}|f|\right)\right\|_{L^{1}(\mu)}\right\}^{q_{0}} .
\end{aligned}
$$


Now we turn our attention to $\mathrm{I}(x)$. Denote by $x_{j}$ the center of $Q_{j}$. Let $\theta$ be a bounded function with $\|\theta\|_{L^{q_{0}^{\prime}(\mu)}} \leq 1$ and the support contained in $\mathbb{R}^{d} \backslash \bigcup_{j} 2 Q_{j}$. Write

$$
\begin{aligned}
& \left|\int_{\mathbb{R}^{d} \backslash \bigcup_{j} 2 Q_{j}} \mathrm{I}(x) \theta(x) d \mu(x)\right| \\
& \leq \sum_{j} \int_{\mathbb{R}^{d} \backslash 2 R_{j}} \int_{\mathbb{R}^{d}}\left|b_{1}^{(j)}(x) \theta(x)\right|\left|\frac{1}{|x-y|^{n-\alpha}}-\frac{1}{\left|x-x_{j}\right|^{n-\alpha}}\right|\left|h_{j}(y)\right| d \mu(y) d \mu(x) \\
& \quad+\sum_{j} \int_{2 R_{j} \backslash 2 Q_{j}}\left|b_{1}^{(j)}(x) \theta(x)\right|\left|I_{\alpha} h_{j}(x)\right| d \mu(x) \\
& \leq C \sum_{j} l\left(Q_{j}\right) \int_{\mathbb{R}^{d}} \int_{\mathbb{R}^{d} \backslash 2 Q_{j}} \frac{\left|b_{1}^{(j)}(x) \theta(x)\right|}{\left|x-x_{j}\right|^{n+1-\alpha}}\left|h_{j}(y)\right| d \mu(y) d \mu(x) \\
& \quad+\sum_{j} \int_{2 R_{j} \backslash 2 Q_{j}}\left|b_{1}^{(j)}(x) \theta(x)\right|\left|I_{\alpha}\left(w_{j} f\right)(x)\right| d \mu(x) \\
& \quad+\sum_{j} \int_{2 R_{j}}\left|b_{1}^{(j)}(x) \theta(x)\right|\left|I_{\alpha} \phi_{j}(x)\right| d \mu(x) \\
& =\mathrm{G}+\mathrm{H}+\mathrm{J} .
\end{aligned}
$$

Invoking the condition (1.1), we have

$$
\begin{aligned}
& \int_{\mathbb{R}^{d} \backslash 2 Q_{j}} \frac{\left|b_{1}^{(j)}(x) \theta(x)\right|}{\left|x-x_{j}\right|^{n+1-\alpha}} d \mu(x) \\
& \leq C \sum_{k=1}^{\infty}\left[2^{k} l\left(Q_{j}\right)\right]^{-n-1+\alpha} \int_{2^{k+1} Q_{j}}\left|b_{1}(x)-m_{2^{\widehat{k+1} Q_{j}}}\left(b_{1}\right)\right||\theta(x)| d \mu(x) \\
& \quad+C \sum_{k=1}^{\infty}\left[2^{k} l\left(Q_{j}\right)\right]^{-n-1+\alpha}\left|m_{\widetilde{Q_{j}}}\left(b_{1}\right)-m_{2^{\left({ }^{k+1} Q_{j}\right.}}\left(b_{1}\right)\right| \int_{2^{k} Q_{j}} \theta(x) d \mu(x) \\
& \leq C \sum_{k=1}^{\infty}\left[2^{k} l\left(Q_{j}\right)\right]^{-n-1+\alpha}\left[\int_{2^{k+1} Q_{j}}\left|b_{1}(x)-m_{2^{k+1} Q_{j}}\left(b_{1}\right)\right|^{q_{0}} d \mu(x)\right]^{1 / q_{0}} \\
& \quad+C \sum_{k=1}^{\infty} K_{2^{k+1} Q_{j}, Q_{j}}\left[2^{k} l\left(Q_{j}\right)\right]^{-n-1+\alpha} \mu\left(2^{k+1} Q_{j}\right)^{1 / q_{0}} \\
& \leq C \sum_{k=1}^{\infty}\left[2^{k} l\left(Q_{j}\right)\right]^{-n-1+\alpha} \mu\left(2^{k+2} Q_{j}\right)^{1 / q_{0}}+C l\left(Q_{j}\right)^{-1} \\
& \leq C l\left(Q_{j}\right)^{-1} .
\end{aligned}
$$


Therefore,

(3.8) $\quad \mathrm{G} \leq C \sum_{j}\left\|h_{j}\right\|_{L^{1}(\mu)} \leq C \sum_{j} \int_{Q_{j}}|f(y)| d \mu(y) \leq C \int_{\mathbb{R}^{d}}|f(y)| d y$,

which is a desired estimate for G.

On the other hand, applying the Hölder inequality and the $\left(L^{p_{1}}, L^{q_{1}}\right)$ boundedness of $I_{\alpha}$ (see Proposition 6.2 in [6]), we obtain

$$
\begin{aligned}
\mathrm{J} \leq & \sum_{j} \int_{2 R_{j}}\left|b_{1}(x)-m_{\widetilde{2 R_{j}}}\left(b_{1}\right)\right|\left|I_{\alpha} \phi_{j}(x) \theta(x)\right| d \mu(x) \\
& +\sum_{j}\left|m_{\widetilde{Q_{j}}}\left(b_{1}\right)-m_{\widetilde{2 R_{j}}}\left(b_{1}\right)\right| \int_{2 R_{j}}\left|I_{\alpha} \phi_{j}(x)\right| \theta(x)|| d \mu(x) \\
\leq & \|\theta\|_{L^{q_{0}^{\prime}}(\mu)}\left\{\sum_{j}\left[\int_{2 R_{j}}\left|b_{1}(x)-m_{\widetilde{2 R_{j}}}\left(b_{1}\right)\right|^{q_{0}}\left|I_{\alpha} \phi_{j}(x)\right|^{q_{0}} d \mu(x)\right]^{1 / q_{0}}\right. \\
& \left.+\sum_{j}\left[\int_{2 R_{j}}\left|I_{\alpha} \phi_{j}(x)\right|^{q_{0}} d \mu(x)\right]^{1 / q_{0}}\left|m_{\widetilde{Q_{j}}}\left(b_{1}\right)-m_{\widetilde{2 R_{j}}}\left(b_{1}\right)\right|\right\} \\
\leq & \sum_{j}\left[\int_{2 R_{j}}\left|b_{1}(x)-m_{\widetilde{2 R_{j}}}\left(b_{1}\right)\right|^{q_{0}\left(q_{1} / q_{o}\right)^{\prime}} d \mu(x)\right]^{1 / q_{0}-1 / q_{1}}\left\|I_{\alpha} \phi_{j}\right\|_{L^{q_{1}}(\mu)} \\
& +\sum_{j}\left[\mu\left(4 R_{j}\right)\right]^{1 / q_{0}-1 / q_{1}}\left\|I_{\alpha} \phi_{j}\right\|_{L^{q_{1}}(\mu)}\left|m_{\widetilde{Q_{j}}}\left(b_{1}\right)-m_{\widetilde{2 R_{j}}}\left(b_{1}\right)\right| \\
\leq & C \sum_{j}\left[\mu\left(4 R_{j}\right)\right]^{1 / q_{0}-1 / q_{1}}\left\|I_{\alpha} \phi_{j}\right\|_{L^{q_{1}}(\mu)}\left[1+\left|m_{\widetilde{Q_{j}}}(b)-m_{\widetilde{2 R_{j}}}\left(b_{1}\right)\right|\right] \\
\leq & C \sum_{j}\left[\mu\left(4 R_{j}\right)\right]^{1 / q_{0}-1 / q_{1}}\left\|\phi_{j}\right\|_{L^{p_{1}}(\mu)} \\
\leq & C \sum_{j}\left[\mu\left(4 R_{j}\right)\right]^{1 / q_{0}-1 / q_{1}}\left\|\phi_{j}\right\|_{L^{\infty}(\mu)}\left[\mu\left(R_{j}\right)\right]^{1 / p_{1}} \\
\leq & \int_{\mathbb{R}^{d}}|f(y)| d \mu(y),
\end{aligned}
$$


where we have used the estimate that

$\left|m_{\widetilde{Q_{j}}}\left(b_{1}\right)-m_{\widetilde{2 R_{j}}}\left(b_{1}\right)\right| \leq\left|m_{\widetilde{Q_{j}}}\left(b_{1}\right)-m_{R_{j}}\left(b_{1}\right)\right|+\left|m_{R_{j}}\left(b_{1}\right)-m_{\widetilde{2 R_{j}}}\left(b_{1}\right)\right| \leq C$,

by recalling that $R_{j}$ is $\left(2, \beta_{d}\right)$-doubling and $\widetilde{R_{j}}=R_{j}$.

To estimate $\mathrm{H}$, observe that for $x \in 2 R_{j} \backslash 2 Q_{j}$,

$$
\left|I_{\alpha}\left(w_{j} f\right)(x)\right| \leq C \frac{1}{\left|x-x_{j}\right|^{n-\alpha}} \int_{Q_{j}}|f(y)| d \mu(y) .
$$

Write

$$
\begin{aligned}
& \mathrm{H} \leq C \sum_{j}\left\{\int_{2 R_{j} \backslash R_{j}} \frac{\left|b_{1}^{(j)}(x) \theta(x)\right|}{\left|x-x_{j}\right|^{n-\alpha}} d \mu(x)\right. \\
&\left.\quad+\int_{R_{j} \backslash Q_{j}} \frac{\left|b_{1}^{(j)}(x) \theta(x)\right|}{\left|x-x_{j}\right|^{n-\alpha}} d \mu(x)\right\} \int_{Q_{j}}|f(y)| d \mu(y) \\
& \leq C \sum_{j} \frac{1}{l\left(R_{j}\right)^{n-\alpha}}\|\theta\|_{L^{q_{0}^{\prime}}(\mu)}\left\{\int_{2 R_{j}}\left|b_{1}^{(j)}(x)\right|^{q_{0}} d \mu(x)\right\}^{1 / q_{0}} \int_{Q_{j}}|f(y)| d \mu(y) \\
&+C \sum_{j} \sum_{k=0}^{N-1} l\left(6^{k} Q_{j}\right)^{-n+\alpha} \int_{6^{k+1} Q_{j} \backslash 6^{k} Q_{j}} b_{1}(x)-m_{6^{k+1} Q_{j}}\left(b_{1}\right)|| \theta(x) \mid d \mu(x) \\
&+C \sum_{j} \sum_{k=0}^{N-1} l\left(6^{k} Q_{j}\right)^{-n+\alpha}\left|m_{\widetilde{Q_{j}}}\left(b_{1}\right)-m_{6^{k+1} Q_{j}}\left(b_{1}\right)\right| \\
& \times \int_{Q_{j}^{k+1} Q_{j}}|\theta(x)| d \mu(x) \mid d \mu(y) \\
& \times \int_{Q_{j}}|f(y)| d \mu(y),
\end{aligned}
$$

where $N$ is the positive integer such that $R_{j}=6^{N} Q_{j}$. Obviously, for each $0 \leq k \leq N-1,6^{k+1} Q_{j} \subset R_{j}$ and so

$$
\left|m_{\widetilde{Q_{j}}}\left(b_{1}\right)-m_{6^{k+1} Q_{j}}\left(b_{1}\right)\right| \leq C K_{Q_{j}, 6^{k+1} Q_{j}} \leq C K_{Q_{j}, R_{j}} \leq C,
$$


which leads to that

$$
\begin{aligned}
\mathrm{H} \leq & C \sum_{j} \int_{Q_{j}}|f(y)| d \mu(y) \\
& +C \sum_{j} \sum_{k=0}^{N-1} l\left(6^{k} Q_{j}\right)^{-n+\alpha}\left[\mu\left(2 \times 6^{k+1} Q_{j}\right)\right]^{1 / q_{0}} \int_{Q_{j}}|f(y)| d \mu(y) \\
& +C \sum_{j} \sum_{k=0}^{N-1} l\left(6^{k} Q_{j}\right)^{-n+\alpha}\left[\mu\left(6^{k+1} Q_{j}\right)\right]^{1 / q_{0}} \int_{Q_{j}}|f(y)| d \mu(y) \\
\leq & C \sum_{j}\left\{1+\sum_{k=0}^{N-1} l\left(6^{k} Q_{j}\right)^{-n+\alpha}\left[\mu\left(2 \times 6^{k+1} Q_{j}\right)\right]^{1 / q_{0}}\right\} \int_{Q_{j}}|f(y)| d \mu(y) .
\end{aligned}
$$

Note that there is no $\left(6,6^{n+1}\right)$-doubling cube between $Q_{j}$ and $R_{j}$. It follows that for each integer $k$ with $0 \leq k \leq N-1$,

$$
\begin{aligned}
\mu\left(6^{k+1} Q_{j}\right) & \leq \frac{\mu\left(6^{N} Q_{j}\right)}{6^{(n+1)(N-k-1)}} \\
& \leq C l\left(6^{N} Q_{j}\right)^{n} 6^{-(n+1)(N-k-1)} \\
& =C l\left(6^{k} Q_{j}\right)^{n} 6^{k-N} .
\end{aligned}
$$

We thus obtain that for each fixed $j$,

$$
\begin{aligned}
& \sum_{k=0}^{N-1} l\left(6^{k} Q_{j}\right)^{-n+\alpha}\left[\mu\left(6^{k+2} Q_{j}\right)\right]^{1 / q_{0}} \leq C \sum_{k=1}^{N-1} l\left(6^{k-1} Q_{j}\right)^{-n+\alpha}\left[\mu\left(6^{k+1} Q_{j}\right)\right]^{1 / q_{0}} \\
&+l\left(6^{N-1} Q_{j}\right)^{-n+\alpha}\left[\mu\left(6 R_{j}\right)\right]^{1 / q_{0}} \\
& \leq C \sum_{k=1}^{\infty} 6^{-k / q_{0}}+C \\
& \leq C
\end{aligned}
$$

Thus,

$$
\mathrm{H} \leq C \sum_{j} \int_{Q_{j}}|f(y)| d \mu(y) \leq C \int_{\mathbb{R}^{d}}|f(y)| d \mu(y) .
$$


Combining the estimates (3.8), (3.9) and (3.10) above yields

$\left|\int_{\mathbb{R}^{d} \backslash \bigcup_{j} 2 Q_{j}} \mathrm{I}(x) \theta(x) d \mu(x)\right| \leq C \sum_{j} \int_{Q_{j}}|f(y)| d \mu(y) \leq C \int_{\mathbb{R}^{d}}|f(y)| d \mu(y)$,

and so

$$
\begin{aligned}
\mu\left(\left\{x \in \mathbb{R}^{d} \backslash \bigcup_{j} 2 Q_{j}:|\mathrm{I}(x)|>\lambda\right\}\right) & \leq C \lambda^{-q_{0}} \int_{\mathbb{R}^{d} \backslash \bigcup_{j} 2 Q_{j}}|\mathrm{I}(x)|^{q_{0}} d \mu(x) \\
& \leq C\left[\lambda^{-1} \int_{\mathbb{R}^{d}}|f(y)| d \mu(y)\right]^{q_{0}} .
\end{aligned}
$$

The estimates (3.7) and (3.11) then yield (3.4), and we have completed the proof of the theorem for $m=1$.

Step II: In this step, we prove Theorem 1.2 for all $m \in \mathbb{N}$ by induction on $m$. To this end, we assume that $m \geq 2$ is an integer and that for any $1 \leq i \leq m-1$ and any subset $\sigma=\{\sigma(1), \ldots, \sigma(i)\}$ of $\{1, \ldots, m\}$, Theorem 1.2 is true. We now verify that Theorem 1.2 is also true for $m$.

For each fixed $f$ and $\lambda>0$, we decompose $f$ by the same way as in Step I; see (I), (II) and (III) in the proof of Step I. And let $Q_{j}, R_{j}, \phi_{j}$, $w_{j}, g, h$ be the same as in Step I. To prove the theorem in this case, it suffices to verity

$$
\begin{aligned}
\mu(\{x \in & \left.\left.\mathbb{R}^{d} \backslash \bigcup_{j} 2 Q_{j}:\left|I_{\alpha ; b_{1}, \ldots, b_{m}} h(x)\right|>\lambda\right\}\right) \\
& \leq C\left\{\sum_{i=0}^{\infty} \sum_{\sigma \in C_{i}^{m}} \Phi_{1 / r_{\sigma^{\prime}}}\left[\left\|\Phi_{1 / r_{\sigma}}\left(\lambda^{-1}|f|\right)\right\|_{L^{1}(\mu)}\right]\right\}^{q_{0}},
\end{aligned}
$$

where for $\sigma \in C_{i}^{m}, 1 / r_{\sigma}=1 / r_{\sigma(1)}+\cdots+1 / r_{\sigma(1)}$ and $1 / r_{\sigma^{\prime}}=1 / r-1 / r_{\sigma}$.

For simplicity, we first introduce some notation. Let $\vec{b}=\left\{b_{1}, \ldots, b_{m}\right\}$. For all $1 \leq i \leq m$ and $\sigma=\{\sigma(1), \ldots, \sigma(i)\} \in C_{i}^{m}$, we define $\vec{b}_{\sigma}=$ $\left\{b_{\sigma(1)}, \ldots, b_{\sigma(i)}\right\}$ and set

$$
I_{\alpha ; b_{\sigma(1)}, \ldots, b_{\sigma(i)}} f(x)=I_{\alpha ; \vec{b}_{\sigma}} f(x) .
$$

In particular, when $\sigma=\{1, \ldots, m\}$, we denote $I_{\alpha ; b_{1}, \ldots, b_{m}}$ simply by $I_{\alpha ; \vec{b}}$. For $1 \leq i \leq m$ and all $\sigma \in C_{i}^{m}$, we write

$$
[b(y)-b(z)]_{\sigma}=\left[b_{\sigma(1)}(y)-b_{\sigma(1)}(z)\right] \times \cdots \times\left[b_{\sigma(i)}(y)-b_{\sigma(i)}(z)\right]
$$


and

$$
\left[m_{\widetilde{Q}}(b)-b(y)\right]_{\sigma}=\left[m_{\widetilde{Q}}\left(b_{\sigma(1)}\right)-b_{\sigma(1)}(y)\right] \times \cdots \times\left[m_{\widetilde{Q}}\left(b_{\sigma(i)}\right)-b_{\sigma(i)}(y)\right],
$$

where $Q$ is any cube in $\mathbb{R}^{d}$ and $y, z \in \mathbb{R}^{d}$. With the aid of the formula

$$
\prod_{i=1}^{m}\left[m_{\widetilde{Q}}\left(b_{i}\right)-b_{i}(z)\right]=\sum_{i=0}^{m} \sum_{\sigma \in C_{i}^{m}}[b(y)-b(z)]_{\sigma^{\prime}}\left[m_{\widetilde{Q}}(b)-b(y)\right]_{\sigma}
$$

for $y, z \in \mathbb{R}^{d}$, where if $i=0$, then $\sigma^{\prime}=\{1, \ldots, m\}$ and $\sigma=\emptyset$, it is easy to see

$$
\begin{aligned}
I_{\alpha ; \vec{b}} h(x)= & \sum_{j} \sum_{i=1}^{m}\left[m_{\widetilde{Q}_{j}}\left(b_{i}\right)-b_{i}(x)\right] I_{\alpha} h_{j}(x) \\
& -\sum_{j} I_{\alpha}\left(\prod_{i=1}^{m}\left[m_{\widetilde{Q}_{j}}\left(b_{i}\right)-b_{i}\right] h_{j}\right)(x) \\
& -\sum_{j} \sum_{i=1}^{m-1} \sum_{\sigma \in C_{i}^{m}} I_{\alpha ; \vec{b}_{\sigma^{\prime}}}\left(\left[m_{\widetilde{Q}_{j}}(b)-b\right]_{\sigma} h_{j}\right)(x) \\
= & I_{\alpha ; \vec{b}}^{\mathrm{I}} h(x)-I_{\alpha ; \vec{b}}^{\mathrm{II}} h(x)-\sum_{i=1}^{m-1} \sum_{\sigma \in C_{i}^{m}} I_{\alpha ; \vec{b}_{\sigma^{\prime}}}^{\mathrm{III}} h(x) .
\end{aligned}
$$

An argument similar to that for I $(x)$ in Step I gives us that

$$
\mu\left(\left\{x \in \mathbb{R}^{d} \backslash \bigcup_{j} 2 Q_{j}:\left|I_{\alpha ; \vec{b}}^{\mathrm{I}} h(x)\right|>\lambda\right\}\right) \leq C\left\{\lambda^{-1} \int_{\mathbb{R}^{d}}|f(y)| d \mu(y)\right\}^{q_{0}}
$$

and an argument similar to that for $\mathrm{I} I(x)$ as in Step I yields

$$
\begin{aligned}
& \mu\left(\left\{x \in \mathbb{R}^{d} \backslash \bigcup_{j} 2 Q_{j}:\left|I_{\alpha ; \dot{b}}^{\mathrm{II}} h(x)\right|>\lambda\right\}\right) \\
& \quad \leq C\left\{\Phi_{1 / r}\left(\lambda^{-1}\|f\|_{L^{1}(\mu)}\right)+\left\|\Phi_{1 / r}\left(\lambda^{-1}|f|\right)\right\|_{L^{1}(\mu)}\right\}^{q_{0}}
\end{aligned}
$$


Now we estimate $I_{\alpha ; \vec{b}_{\sigma^{\prime}}}^{\mathrm{III}} h(x)$. For each fixed $i$ with $1 \leq i \leq m-1$, the induction hypothesis now states that

$$
\begin{aligned}
& \mu\left(\left\{x \in \mathbb{R}^{d}:\left|I_{\alpha ; \vec{b}_{\sigma^{\prime}}}^{\mathrm{III}} h(x)\right|>\lambda\right\}\right) \\
& \leq C\left\{\sum_{l=0}^{m-i} \sum_{\eta \in C_{l}^{m-i}} \Phi_{1 / r_{\eta^{\prime}}}\left[\left\|\Phi_{1 / r_{\eta}}\left(\lambda^{-1} \sum_{j}\left|\left[m_{\widetilde{Q}_{j}}(b)-b\right]_{\sigma} h_{j}\right|\right)\right\|_{L^{1}(\mu)}\right]\right\}^{q_{0}} \\
& \leq C\left\{\sum_{l=1}^{m-i} \sum_{\eta \in C_{l}^{m-i}} \Phi_{1 / r_{\eta^{\prime}}}\left[\left\|\Phi_{1 / r_{\eta}}\left(\lambda^{-1} \sum_{j}\left|\left[m_{\widetilde{Q}_{j}}(b)-b\right]_{\sigma} f \omega_{j}\right|\right)\right\|_{L^{1}(\mu)}\right]\right\}^{q_{0}} \\
& +C\left\{\sum_{l=1}^{m-i} \sum_{\eta \in C_{l}^{m-i}} \Phi_{1 / r_{\eta^{\prime}}}\left[\left\|\Phi_{1 / r_{\eta}}\left(\lambda^{-1} \sum_{j}\left|\left[m_{\widetilde{Q}_{j}}(b)-b\right]_{\sigma} \phi_{j}\right|\right)\right\|_{L^{1}(\mu)}\right]\right\}^{q_{0}} \\
& +C\left\{\Phi_{1 / r_{\sigma^{\prime}}}\left[\lambda^{-1} \sum_{j}\left[m_{\widetilde{Q}_{j}}(b)-b\right]_{\sigma} h_{j}\|\|_{L^{1}(\mu)}\right]\right\}^{q_{0}} \\
& =\mathrm{A}+\mathrm{B}+\mathrm{D} .
\end{aligned}
$$

For A, we consider the following two cases.

Case I. $\lambda \geq 1$. Set $\Psi_{r}(t)=\exp t^{r}-1$. Note

$$
\begin{aligned}
& \Psi_{r}^{-1}(t) \sim \log ^{1 / r}(2+t), \\
& \Phi_{r}^{-1}(t) \sim t \log ^{-1 / r}(2+t) .
\end{aligned}
$$

By Lemma 2.2 in [16], we see that for any $1 \leq i \leq m-1, \sigma \in C_{i}^{m}$ and any $t_{0}, t_{1}, \ldots, t_{i}>0$,

$$
\Phi_{1 / r_{\eta}}\left(t_{0} t_{1} \cdots t_{i}\right) \leq C\left[\Phi_{1 / r_{\eta}+1 / r_{\sigma}}\left(t_{0}\right)+\Psi_{r_{\sigma(1)}}\left(t_{1}\right)+\cdots+\Psi_{r_{\sigma(i)}}\left(t_{i}\right)\right] .
$$


From this, it follows that

$$
\begin{aligned}
& \|\left.\Phi_{1 / r_{\eta}}\left(\lambda^{-1} \sum_{j}\left|\left[m_{\widetilde{Q}_{j}}(b)-b\right]_{\sigma} f \omega_{j}\right|\right)\right|_{L^{1}(\mu)} \\
\leq & C \sum_{j} \int_{Q_{j}} \Phi_{1 / r_{\eta}}\left(\lambda^{-1}\left|f(x)\left[m_{\widetilde{Q}_{j}}(b)-b(x)\right]_{\sigma}\right|\right) d \mu(x) \\
\leq & C \sum_{j} \int_{Q_{j}} \Phi_{1 / r_{\eta}+1 / r_{\sigma}}\left(\lambda^{-1}|f(x)|\right) d \mu(x) \\
& +C \sum_{j} \sum_{l=1}^{i} \int_{Q_{j}} \Psi_{r_{\sigma(l)}}\left(\left|m_{\widetilde{Q}_{j}}\left(b_{\sigma(l)}\right)-b_{\sigma(l)}(x)\right|\right) d \mu(x) \\
\leq & C \sum_{j} \int_{Q_{j}} \Phi_{1 / r_{\eta}+1 / r_{\sigma}}\left(\lambda^{-1}|f(x)|\right) d \mu(x)+C \sum_{j} \mu\left(2 Q_{j}\right) \\
\leq & C \sum_{j} \int_{Q_{j}} \Phi_{1 / r_{\eta}+1 / r_{\sigma}}\left(\lambda^{-1}|f(x)|\right) d \mu(x)+C \lambda^{-q_{0}} \sum_{j} \int_{Q_{j}}|f(x)| d \mu(x) \\
\leq & C \sum_{j} \int_{Q_{j}} \Phi_{1 / r_{\eta}+1 / r_{\sigma}}\left(\lambda^{-1}|f(x)|\right) d \mu(x)+C \lambda^{-1} \sum_{j} \int_{Q_{j}}|f(x)| d \mu(x) \\
\leq & C\left\|\Phi_{1 / r_{\eta}+1 / r_{\sigma}}\left(\lambda^{-1}|f(x)|\right)\right\| L_{L^{1}(\mu)} .
\end{aligned}
$$

Case II. $0<\lambda<1$. In this case, we have

$$
\begin{aligned}
& \left\|\Phi_{1 / r_{\eta}}\left(\lambda^{-1} \sum_{j}\left|\left[m_{\widetilde{Q}_{j}}(b)-b\right]_{\sigma} f \omega_{j}\right|\right)\right\|_{L^{1}(\mu)} \\
\leq & C \sum_{j} \int_{Q_{j}} \Phi_{1 / r_{\eta}}\left(\lambda^{q_{0}-1}\right) \Phi_{1 / r_{\eta}}\left(\lambda^{-q_{0}}\left|f(x)\left[m_{\widetilde{Q}_{j}}(b)-b\right]_{\sigma}\right|\right) d \mu(x) \\
\leq & C \sum_{j} \lambda^{q_{0}-1} \int_{Q_{j}} \Phi_{1 / r_{\eta}+1 / r_{\sigma}}\left(\lambda^{-q_{0}}|f(x)|\right) d \mu(x)+C \sum_{j} \lambda^{q_{0}-1} \mu\left(2 Q_{j}\right) \\
\leq & C \sum_{j} \int_{Q_{j}} \lambda^{-1}|f(x)| \log ^{1 / r_{\eta}+1 / r_{\sigma}}\left(2+\lambda^{-q_{0}}|f(x)|\right) d \mu(x) \\
& +C \lambda^{-1} \sum_{j} \int_{Q_{j}}|f(x)| d \mu(x) \\
\leq & C\left\|\Phi_{1 / r_{\eta}+1 / r_{\sigma}}\left(\lambda^{-1}|f(x)|\right)\right\|_{L^{1}(\mu)}+\Phi_{1 / r_{\eta}+1 / r_{\sigma}}\left(\lambda^{-1}\|f\|_{L^{1}(\mu)}\right) .
\end{aligned}
$$


Thus we always have

$$
\begin{array}{r}
\mathrm{A} \leq C\left\{\sum_{l=1}^{m-i} \sum_{\eta \in C_{l}^{m-i}} \Phi_{1 / r_{\eta^{\prime}}}\left(\left\|\Phi_{1 / r_{\eta}+1 / r_{\sigma}}\left(\lambda^{-1}|f(x)|\right)\right\|_{L^{1}(\mu)}\right)\right. \\
\left.+\Phi_{1 / r}\left(\lambda^{-1}\|f\|_{L^{1}(\mu)}\right)\right\}^{q_{0}} .
\end{array}
$$

Set $s_{j}(x)=\lambda^{-q_{0}}\left|\phi_{j}(x)\right|$ and denote by $\Lambda$ a finite subset of $\mathbb{N}$. From (I), (II) and (III) of the decomposition of $f,(3.2)$, the convexity of the function $\Phi_{1 / r_{\eta}}(t)$ and the fact that $K_{R_{j}, \widetilde{Q_{j}}} \leq C$, it follows that

$$
\begin{aligned}
& \int_{\mathbb{R}^{d}} \Phi_{1 / r_{\eta}}\left\{\sum_{j \in \Lambda} \frac{\left|\phi_{j}(x)\right|}{\lambda}\left|\left[m_{\widetilde{Q_{j}}}(b)-b(x)\right]_{\sigma}\right| \chi_{R_{j}}(x)\right\} d \mu(x) \\
& \leq C \Phi_{1 / r_{\eta}}\left(\lambda^{q_{0}-1}\right) \int_{\mathbb{R}^{d}} \Phi_{1 / r_{\eta}}\left(\sum_{l \in \Lambda} s_{l}(x)\right) \Phi_{1 / r_{\eta}} \\
& \times\left[\sum_{j \in \Lambda}\left(\frac{s_{j}(x)}{\sum_{l \in \Lambda} s_{l}(x)}\right)\left|\left[m_{\widetilde{Q_{j}}}(b)-b(x)\right]_{\sigma}\right| \chi_{R_{j}}(x)\right] d \mu(x) \\
& \leq C \Phi_{1 / r_{\eta}}\left(\lambda^{q_{0}-1}\right) \sum_{j \in \Lambda} \int_{\mathbb{R}^{d}} \Phi_{1 / r_{\eta}}\left(\sum_{l \in \Lambda} s_{l}(x)\right)\left(\sum_{l \in \Lambda} s_{l}(x)\right)^{-1} s_{j}(x) \\
& \times \Phi_{1 / r_{\eta}}\left\{\left|\left[m_{\widetilde{Q_{j}}}(b)-b(x)\right]_{\sigma}\right| \chi_{R_{j}}(x)\right\} d \mu(x) \\
& \leq C \Phi_{1 / r_{\eta}}\left(\lambda^{q_{0}-1}\right) \sum_{j \in \Lambda} \int_{R_{j}} s_{j}(x)\left|\left[m_{\widetilde{Q_{j}}}(b)-b(x)\right]_{\sigma}\right|^{2} d \mu(x) \\
& \leq C \Phi_{1 / r_{\eta}}\left(\lambda^{q_{0}-1}\right) \sum_{j \in \Lambda} \frac{\left\|\phi_{j}\right\|_{L^{\infty}(\mu)}}{\lambda^{q_{0}}} \mu\left(R_{j}\right)\left[1+\left(K_{R_{j}, \widetilde{Q_{j}}}\right)^{2}\right] \\
& \leq C \Phi_{1 / r_{\eta}}\left(\lambda^{q_{0}-1}\right) \sum_{j \in \Lambda} \frac{\left\|\phi_{j}\right\|_{L^{\infty}(\mu)}}{\lambda^{q_{0}}} \mu\left(R_{j}\right) \\
& \leq C \sum_{j \in \Lambda} \frac{1}{\lambda} \log ^{1 / r_{\eta}}\left(2+\lambda^{q_{0}-1}\right)\left\|\phi_{j}\right\|_{L^{\infty}(\mu)} \mu\left(R_{j}\right) \\
& \leq C \sum_{j \in \Lambda} \mu\left(Q_{j}\right) \frac{1}{\mu\left(Q_{j}\right)} \int_{Q_{j}} \frac{|f(x)|}{\lambda} d \mu(x) \log ^{1 / r_{\eta}}\left(2+\frac{1}{\mu\left(Q_{j}\right)} \int_{Q_{j}} \frac{|f(x)|}{\lambda} d \mu(x)\right) \\
& =C \sum_{j \in \Lambda} \mu\left(Q_{j}\right) \Phi_{1 / r_{\eta}}\left[\frac{1}{\mu\left(Q_{j}\right)} \int_{Q_{j}} \frac{|f(x)|}{\lambda} d \mu(x)\right] \\
& \leq C \int_{\mathbb{R}^{d}} \frac{|f(x)|}{\lambda} \log ^{1 / r_{\eta}}\left(2+\frac{|f(x)|}{\lambda}\right) d \mu(x)=C\left\|\Phi_{1 / r_{\eta}}\left(\lambda^{-1}|f|\right)\right\|_{L^{1}(\mu)},
\end{aligned}
$$


where in the second-to-last step, we used the Jenson inequality, and the constant $C$ is independent of $\Lambda$. From the arbitrariness of $\Lambda$, it follows that

$\left\|\Phi_{1 / r_{\eta}}\left(\lambda^{-1} \sum_{j}\left|\left[m_{\widetilde{Q}_{j}}(b)-b\right]_{\sigma} \phi_{j}\right|\right)\right\|_{L^{1}(\mu)} \leq C\left\|\Phi_{1 / r_{\eta}}\left(\lambda^{-1}|f|\right)\right\|_{L^{1}(\mu)}$.

Thus,

$$
\mathrm{B} \leq C\left\{\sum_{l=1}^{m-i} \sum_{\eta \in C_{l}^{m-i}} \Phi_{1 / r_{\eta^{\prime}}}\left(\left\|\Phi_{1 / r_{\eta}}\left(\lambda^{-1}|f|\right)\right\|_{L^{1}(\mu)}\right)\right\}^{q_{0}} .
$$

An argument similar to that for $I I(x)$ as in Step I yields

$$
\begin{aligned}
\mathrm{D} \leq C & \left\{\Phi_{1 / r_{\sigma^{\prime}}}\left[\Phi_{1 / r_{\sigma}}\left(\lambda^{-1}\|f\|_{L^{1}(\mu)}\right)\right]\right. \\
& \left.+\Phi_{1 / r_{\sigma^{\prime}}}\left(\left\|\Phi_{1 / r_{\sigma}}\left(\lambda^{-1}|f|\right)\right\|_{L^{1}(\mu)}\right)\right\}^{q_{0}} \\
\leq & C\left\{\Phi_{1 / r}\left(\lambda^{-1}\|f\|_{L^{1}(\mu)}\right)+\Phi_{1 / r_{\sigma^{\prime}}}\left(\left\|\Phi_{1 / r_{\sigma}}\left(\lambda^{-1}|f|\right)\right\|_{L^{1}(\mu)}\right)\right\}^{q_{0}} .
\end{aligned}
$$

Combining with (3.15), (3.16) and (3.17) tells us that

$$
\begin{aligned}
& \mu\left(\left\{x \in \mathbb{R}^{d}:\left|I_{\alpha ; \vec{b}_{\sigma^{\prime}}}^{\mathrm{III}} h(x)\right|>\lambda\right\}\right) \\
& \leq C\left\{\sum_{l=0}^{m-i} \sum_{\eta \in C_{l}^{m-i}} \Phi_{1 / r_{\eta^{\prime}}}\left(\left\|\Phi_{1 / r_{\eta}+1 / r_{\sigma}}\left(\lambda^{-1}|f(x)|\right)\right\|_{L^{1}(\mu)}\right)\right. \\
& \left.+\Phi_{1 / r}\left(\lambda^{-1}\|f\|_{L^{1}(\mu)}\right)\right\}^{q_{0}} .
\end{aligned}
$$

Finally, the estimates (3.13), (3.14) and (3.18) tell us the estimate (3.12), and we have completed the proof of Theorem 1.2.

Acknowledgements. This research was supported by NNSF (No. 10271015) and RFDP (No. 20020027004) of China.

The authors want to express their thanks to Professor Wengu Chen for his providing the authors his paper [2] and to the referee for his very careful reading and many valuable remarks which made this article more readable.

\section{References}

[1] S. Chanillo, A note on commutators, Indiana Univ. Math. J. 31(1) (1982), 7-16. 
[2] W. Chen and E. SaWyer, A note on commutators of fractional integrals with $\operatorname{RBMO}(\mu)$ functions, Illinois J. Math. 46(4) (2002), 1287-1298.

[3] Y. Ding, S. Lu And P. Zhang, Weak estimates for commutators of fractional integral operators, Sci. China Ser. A 44(7) (2001), $877-888$.

[4] J. García-Cuerva and A. E. Gatto, Boundedness properties of fractional integral operators associated to non-doubling measures, Studia Math. 162(3) (2004), 245-261.

[5] J. García-Cuerva and A. E. Gatto, Lipschitz spaces and Calderón-Zygmund operators associated to non-doubling measures, Preprint (2002).

[6] J. García-Cuerva and J. M. Martell, Two-weight norm inequalities for maximal operators and fractional integrals on non-homogeneous spaces, Indiana Univ. Math. J. 50(3) (2001), 1241-1280.

[7] G. Hu, Y. Meng and D. Yang, Multilinear commutators of singular integrals with non doubling measures, Integral Equations $O p$ erator Theory (to appear).

[8] J. Mateu, P. Mattila, A. Nicolau and J. Orobitg, BMO for nondoubling measures, Duke Math. J. 102(3) (2000), 533-565.

[9] Y. Meyer and R. Coifman, "Wavelets. Calderón-Zygmund and multilinear operators", Translated from the 1990 and 1991 French originals by David Salinger, Cambridge Studies in Advanced Mathematics 48, Cambridge University Press, Cambridge, 1997.

[10] F. Nazarov, S. Treil and A. Volberg, Cauchy integral and Calderón-Zygmund operators on nonhomogeneous spaces, Internat. Math. Res. Notices 15 (1997), 703-726.

[11] F. Nazarov, S. Treil and A. Volberg, Weak type estimates and Cotlar inequalities for Calderón-Zygmund operators on nonhomogeneous spaces, Internat. Math. Res. Notices 9 (1998), 463-487.

[12] F. Nazarov, S. Treil and A. Volberg, The $T b$-theorem on non-homogeneous spaces, Acta Math. 190(2) (2003), 151-239.

[13] F. Nazarov, S. Treil and A. VolberG, Accretive system $T b$-theorems on nonhomogeneous spaces, Duke Math. J. 113(2) (2002), 259-312.

[14] J. Orobitg And C. PÉRez, $A_{p}$ weights for nondoubling measures in $\mathbb{R}^{n}$ and applications, Trans. Amer. Math. Soc. 354(5) (2002), 2013-2033 (electronic). 
[15] C. PÉrez And G. Pradolini, Sharp weighted endpoint estimates for commutators of singular integrals, Michigan Math. J. 49(1) (2001), 23-37.

[16] C. PÉrez and R. Trujillo-González, Sharp weighted estimates for multilinear commutators, J. London Math. Soc. (2) 65(3) (2002), 672-692.

[17] X. Tolsa, Cotlar's inequality without the doubling condition and existence of principal values for the Cauchy integral of measures, $J$. Reine Angew. Math. 502 (1998), 199-235.

[18] X. Tolsa, $L^{2}$-boundedness of the Cauchy integral operator for continuous measures, Duke Math. J. 98(2) (1999), 269-304.

[19] X. Tolsa, A T(1) theorem for non-doubling measures with atoms, Proc. London Math. Soc. (3) 82(1) (2001), 195-228.

[20] X. TolsA, BMO, $H^{1}$, and Calderón-Zygmund operators for non doubling measures, Math. Ann. 319(1) (2001), 89-149.

[21] X. Tolsa, Littlewood-Paley theory and the $T(1)$ theorem with non-doubling measures, Adv. Math. 164(1) (2001), 57-116.

[22] X. TolsA, The space $H^{1}$ for nondoubling measures in terms of a grand maximal operator, Trans. Amer. Math. Soc. 355(1) (2003), 315-348 (electronic).

[23] X. Tolsa, A proof of the weak $(1,1)$ inequality for singular integrals with non doubling measures based on a Calderón-Zygmund decomposition, Publ. Mat. 45(1) (2001), 163-174.

Guoen $\mathrm{Hu}$

Department of Applied Mathematics

University of Information Engineering

Zhengzhou 450002

People's Republic of China

E-mail address: huguoen@eyou.com

Yan Meng and Dachun Yang:

Department of Mathematics

Beijing Normal University

Beijing 100875

People's Republic of China

E-mail address: mengyan@mail.bnu.edu.cn

E-mail address: dcyang@bnu.edu.cn

Primera versió rebuda el 23 d'abril de 2003, darrera versió rebuda el 5 de novembre de 2003 . 
